\title{
KOMMUNIKATIVE PRAKTIKEN IN TÜRKISCHSTÄMMIGEN KINDER- UND JUGENDGRUPPEN IN MANNHEIM ${ }^{1}$
}

\begin{abstract}
Auf der Grundlage einer ethnographisch-soziolinguistischen Untersuchung gibt der vorliegende Beitrag einen groben Überblick über die türkische Migrantengemeinde in Mannheim, die Tendenzen zur Segregation und Abgrenzung von der übrigen Gesellschaft zeigt, neben einer Orientierung auf erfolgreiche soziale und berufliche Karrieren im nationalen und internationalen Rahmen. Im Mittelpunkt der Beschreibung steht die soziale und sprachliche Entwicklung von Migrantenkindern und Jugendgruppen in Bezug auf ihre Orientierung in die eine oder andere Richtung, wobei die jeweiligen sprachlichen Repertoires und Kommunikationspraktiken kurz geschildert werden.
\end{abstract}

Based on an ethnographic-sociolinguistic study, this paper gives a rough outline of the Turkish migrant Community in Mannheim, Germany, which shows tendencies towards segregation and sealing itself off from mainstream society alongside orientations towards successful social and professional carriers in national and intemational frameworks. The paper focuses on the social and linguistic development of migrant children and youth groups in relation to their orientation in one or the other direction, and gives a brief description of the linguistic repertoires and communicative practices of the two groups.

\section{Gegenstand und Ziel}

Seit der Pisa-Studie ist das schlechte schulische Abschneiden vor allem türkischer Kinder ins öffentliche Bewusstsein gerückt. In einem Artikel in der „Zeit" z.B. werden sie als „Spitzenreiter im (schulischen) Scheitern" bezeichnet ${ }^{2}$ und in dem jüngsten Bildungsbericht von Baden-Württemberg wird Kindern mit Migrationshintergrund, vor allem türkischen Kindern, ein „erheblicher Bildungsrückstand" attestiert. ${ }^{3}$ Für die Bildungsmisere der Kinder werden vor allem zwei Faktoren verantwortlich gemacht: einerseits die geringe Förderung durch die deutsche Schule und andererseits das geringe Bildungsinteresse türkischer Eltern. Doch ein Blick in die Pisa-Ergebnisse anderer Länder zeigt, dass türkische Migrantenkinder dort wesentlich besser abschneiden, ${ }^{4}$ d.h. die türkische Herkunft erklärt nicht, warum die Kinder in deutschen Schulen so wenig erfolgreich sind. F.-O. Radtke und M. Gomolla z.B. sehen das Hauptproblem bei der deutschen Schule und sprechen von einer „,institutionellen Diskriminierung" der Migranten-

1 Der Beitrag ist eine überarbeitete und erweiterte Version von Keim 2004.

2 Vgl. den, Zeit"-Artikel vom 27.02.2003, S.16.

3 Einige Details aus dem Bildungsbericht 2004: Obwohl Migranten nur 12,5\% der Bevölkerung in Ba den-Württemberg ausmachen, ist ihr Anteil in der Sonderschule doppelt so hoch (25\%). Unter den Re alschülem haben nur 7\% der Kinder einen Migrationshintergnund, im Gymnasium sogar nur $4 \%$. In der Hauptschule jedoch stellen sie mit $62 \%$ den größten Anteil, während von den deutschen Schülern nur $24 \%$ die Hauptschule besuchen, zitiert nach einem Artikel im MM vom 9.11.2004, S.5.

4 Vgl. den Artikel in der ,Zeit" Nr. 10, 27.02.2003, S.16. Dort heißt es: ,... ob in Schweden oder Norwe gen, in Österreich oder der Schweiz: Überall können Kinder türkischer Einwanderer besser lesen als hierzulande". 
kinder, da sie aufgrund mangelnder Deutschkenntnisse für die Hauptschule empfohlen werden, auch wenn sie die Fähigkeiten fürs Gymnasium haben. ${ }^{5}$ Und die Erziehungswissenschaftlerin I. Gogolin stellt ganz allgemein fest, dass es der deutschen Schule ,auch nach 40 Jahren Migration" nicht gelungen ist, „Ausländerkindern gleiche Bildungschancen zu geben". ${ }^{6}$

In jüngster Zeit rückt ein weiterer Problembereich in den Fokus einer breiteren öffentlichen Aufmerksamkeit, und zwar die Bildung so genannter „Parallelgesellschaften". Dieser Begriff wird vor allem auf muslimische Migrantengemeinschaften bezogen, die in vielen Städten entstanden sind, und mit Kritik an ihrer Lebensweise verbunden.

Ein mehrjähriges ethnographisch-soziolinguistisch ausgerichtetes Projekt zum Sprachund Kommunikationsverhalten von Migranten der 2. und 3. Generation in Mannheim verschaffte uns Einblick in die ökologischen, kulturellen, sozialen und sprachlichen Verhältnisse unter denen Migrantenfamilien leben und in Entwicklungskarrieren, die ihre Kinder durchlaufen. Nach unseren Erkenntnissen ist die türkische Migrantenpopulation weit ausdifferenziert und umfasst sowohl soziale Formationen, die das Potential einer ethnisch ausgerichteten, von der Mehrheitsgesellschaft abgeschotteten Gemeinschaft in sich tragen, als auch solche, die weltoffen und international agieren und in vielen Lebensbereichen erfolgreich sind. ${ }^{9}$

Im Folgenden werde ich einen Ausschnitt aus der türkischen Migrantenpopulation darstellen, in dem es einerseits Tendenzen zu Segregation und Abschottung, andererseits aber auch gegenläufige Entwicklungen gibt, und werde die unterschiedlichen sozialen und sprachlichen Entwicklungsprozesse und -ergebnisse aufzeigen. Zunächst erfolgt eine Kurzcharakterisierung der untersuchten Migrantengemeinschaft (Kap. 2), dann eine Skizzierung typischer Entwicklungskarrieren der Kinder (Kap. 3) und im Anschluss eine detaillierte Darstellung der Sprach- und Kommunikationspraktiken, die sich im Zusammenhang mit sozialen Leitbildern und Karrieren entwickeln (Kap. 4 und 5).

5 Zitiert nach dem ,Zeit"-Artikel vom 27.10.2003, S. 16.

6 Zitiert nach dem,ZZeit"-Artikel, S. 16.

7 Seit der Ermordung des islam-kritischen Filmemachers Theo van Gogh in den Niederlanden und der anschließenden Kritik an Muslimen, kam der Begriff der „Parallelgesellschaft" in die öffentliche Dis kussion und wurde zur Beschreibung ganz unterschiedlicher Verhältnisse verwendet. Bade z.B. kriti siert in einem Spiegel-Artikel den populistischen Gebrauch des Begriffs und führt folgende Indikatoren für „Parallelgesellschaft" ein: „eine monokulturelle Identität, ein (...) bewusster sozialer Rückzug auch in Siedlung und Lebensalltag, eine weitgehende wirtschaftliche Abgrenzung und eine Doppelung der Institutionen des Staates. Nach dieser Definition könne in Deutschland nicht von Parallelgesellschaften gesprochen werden, sondern nur von sozialem Rückzug"; zitiert nach http://www.spiegel.de/kul-tur/gesellschaft/0,1518,329285,00.html

$8 \mathrm{Zu}$ den Publikationen aus dem Projekt vgl. die Homepage des IDS, Abteilung „Pragmatik", Projekt "deutsch-türkische Sprachvariation".

9 Exemplarisch für eine weltoffene, trans- bzw. internationale Orientienung türkischer Migranten der 2. Generation sind die im Rahmen unseres IDS-Projekts untersuchten Gruppen „Europatürken" und „Un mündige"; vgl. dazu die Beiträge von Aslan und Cindark in diesem Band. Vgl. auch das Sonderheft „Türken in Deutschland - Türken in Europa" Heft 1 (2005) hgg. von isoplan Consult, Saarbrücken, in dem national und international erfolgreiche Karrieren türkischstämmiger Migranten aus verschiedenen Bereichen von Wirtschaft, Politik und Kunst dargestellt werden. 


\section{Charakterisierung der untersuchten Migrantengemeinschaft}

Nach einer Statistik von 2001 haben 21 \% der Mannheimer Bevölkerung einen Migrationshintergrund, und in Mannheim werden derzeit ca. 168 Sprachen gesprochen. Der größte Teil der Migrantinnen ist türkischer Herkunft (34 \%), gefolgt von Italienern (14 $\%$ ), Zuwanderern aus dem ehemaligen „Jugoslawien" ( $8 \%$ ), aus Polen (5\%), Griechenland (5\%), Spanien (3\%) und aus vielen anderen Ländern. ${ }^{10}$ Vor allem zwei Stadtteile der Mannheimer Innenstadt haben einen hohen Anteil nicht-deutschstämmiger Bevölkerung: Der „Jungbusch" mit fast $65 \%$ und der direkt angrenzende Stadtteil „Westliche Unterstadt" mit ca. 47 \% Einwohnern nicht-deutscher Herkunft. Neben der türkischen Gruppe gibt es Migranten slawischer, romanischer, arabischer, afrikanischer und asiatischer Herkunftssprachen. Soziale Netzwerke haben sich fast ausschließlich innerhalb ethnischer Gruppen gebildet, zwischen ethnischen Gruppen gibt es nur wenige Kontakte. In beiden Stadtteilen sind Migrantinnen türkischer Herkunft in der Öffentlichkeit sehr präsent; es gibt ganze Häuserblocks und Straßenzüge, deren Bewohner türkische Namen tragen. Die türkische Migrantengemeinschaft hat eine hohe Infrastruktur entwickelt; es gibt türkische Lebensmittelgeschäfte, Bäckereien, Haushaltswaren- und Kleidergeschäfte, türkische Banken und Immobilienbüros, türkische Friseure, Ärzte und Rechtsanwälte. D.h. für alle Lebensbereiche gibt es türkische Angebote und Dienstleistungen, und man kann in diesem Stadtgebiet über Jahre leben, ohne dass Kontakte zu Deutschen notwendig werden. Aufgrund dieser Infrastruktur und der sozialen Verhältnisse werden die beiden Stadtteile sowohl von Bewohnern als auch von Außenstehenden als „Ghetto" bezeichnet. Eine junge Informantin, die seit ihrer Kindheit dort wohnt, verbindet mit der Bezeichnung „Ghetto" vor allem negative Aspekte:

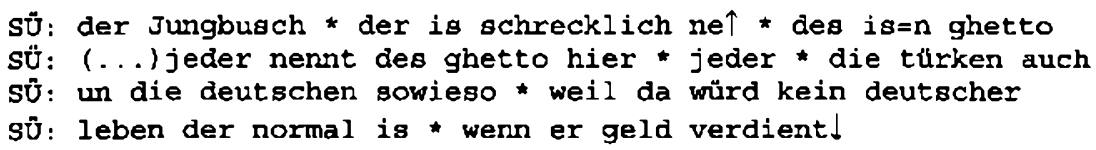

Ähnlich urteilt eine andere junge Migrantin, die den Stadtteil „Jungbusch" mit den amerikanischen Ghettos vergleicht:

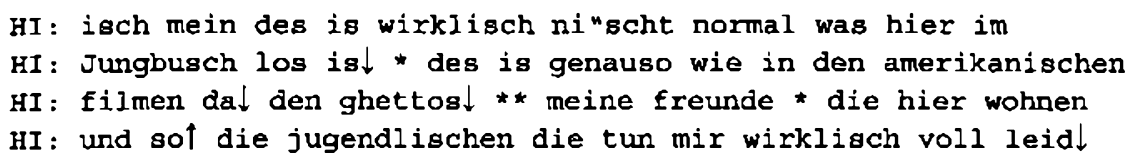

Doch es werden auch positive Aspekte mit dem Leben im Ghetto verbunden. Besonders die erste Migrantengeneration fühlt sich hier wohl und zuhause. Auch viele Jüngere heben die Schutzfunktion des Ghettos und das Gefühl unter gleichen zu leben hervor. Für eine Studentin, die in einem Dorf in Norddeutschland aufwuchs, war der Umzug nach Mannheim und das Eintauchen ins Ghetto entspannend und erleichternd; hier konnte sie sich ungestört und unbeobachtet bewegen. Ähnliche Erfahrungen beschreiben auch andere: Im Ghetto muss man nicht erklären, warum man Kopftuch oder keines trägt, warum man fastet oder warum man noch bei den Eltern lebt. Man muss die sozialen Kon- 
ventionen der türkischen Gemeinschaft nicht rechtfertigen und man muss vor allem nicht beweisen, dass man normal ist. Außerhalb des Ghettos fühlen sich junge Leute ständig aufgefordert, diesen Beweis zu erbringen, da dort jede unbedachte Äußerung dazu führen kann, dass Vorurteile auf Seiten der Deutschen aktiviert werden, gegen die sie sich wehren müssen.

Aufgrund der langjährigen Unsicherheit der Migrationssituation in Deutschland und vieler negativer Erfahrungen durch die deutsche Bevölkerung ${ }^{11}$ hat sich in vielen türkischen Familien eine Art Neoorthodoxie herausgebildet; d.h. diese Familien halten streng an mitgebrachten traditionellen und religiösen Werten und Normen fest, um sich und ihren Kindern Sicherheit zu geben und sie vor deutschen Einflüssen zu schützen. ${ }^{12}$ Das soziale Leben ist in diesen Familien auf das Territorium des Stadtgebiets beschränkt: Verwandte und Freunde leben hier, Einkäufe, Friseur- und Arztbesuche finden in der direkten Nachbarschaft statt, und ein Weg an die Grenze des Ghettos wird - so eine türkische Pädagogin - bereits als Ausland empfunden. Im Leben dieser Ghetto-Netzwerke kommen Deutsche nicht vor und Deutsch hat im Alltag keine Bedeutung. Da die Heiratsmigration in diesen Familien besonders hoch ist, bleiben traditionelle türkische Familienstrukturen erhalten und Türkisch ist auch in der dritten Generation die einzige Familiensprache. ${ }^{13}$

Nach einer neueren Statistik zur Schulsituation in Mannheim (2001) liegt der Anteil von Kindern mit Migrationshintergrund in den beiden Grundschulen des Stadtgebiets mit 77 $90 \%$ sehr hoch. Es gibt Klassen mit Kindern aus 14 Nationen, danunter nur ein oder zwei Deutsche. Diese Situation hat erhebliche Konsequenzen für die Schulkarriere der Kinder: Nur ca. $15 \%$ der Migrantenkinder schaffen den Übergang zu weiterführenden Schulen, obwohl nach Aussage der Lehrenden viele ausgesprochen intelligent sind. Die schulischen Ergebnisse der Hauptschüler sind schlecht: 30-35 \% verlassen die Schule ohne Abschluss, viele mit schlechten Abschlüssen und nur sehr wenigen (14-15\% eines Jahrgangs) gelingt es eine Lehrstelle zu bekommen. Die jugendlichen Hauptschüler haben kaum berufliche Perspektiven und verstehen sich oft auch selbst als loser (Verlierer).

Das zentrale Problem der Kinder bei Schuleintritt ist die geringe deutschsprachige Kompetenz. Da die Deutschprobleme im Regelunterricht nicht abgebaut werden können und es kaum Zusatzprogramme gibt, ${ }^{14}$ vergrößern sie sich im Laufe der Zeit, weil sich der fachliche Stoff ständig ausweitet. Das hat Konsequenzen in allen Fächern, in denen die Kompetenz im Deutschen Voraussetzung ist. Übereinstimmend bezeichnen alle Infor-

11 Vgl. vor allem die rechtliche Unsicherheit, Verlust des Arbeitsplatzes und der

Aufenthaltsgenehmigung

in wirtschaftlichen Krisenzeiten; vgl. auch die ausländer- und besonders türkenfeindlichen Aktionen in den 90er Jahren und die Anschläge auf Türken in Mölln und Solingen.

12 Viele der befragten Jugendlichen berichten z.B., dass ihre Cousins und Cousinen in der Türkei

„freier" lebten als sie hier und deren Eltern viel großzügiger mit Vorschriften und Verboten umgingen.

13 Eine ähnliche Beschreibung türkischer Migrantengemeinschaften in Deutschland findet sich in

Kelek

2005 .

14 Bis vor kurzem gab es in den beiden Grundschulen keine Sprachfördermaßnahmen mehr, da die

dungsbürokratie davon ausging, dass die Kinder in der dritten Generation ganz natürlich Deutsch lemen würden. Seit zwei Jahren gibt es aufgrund einer außerschulischen Initiative Förderunterricht für Erst klässler, seit Ende 2004 auch für Viertklässler. 
mantInnen die Rechtschreibung als katastrophal, einige Lehrerinnen haben ihre Bemühungen in diesem Bereich bereits aufgegeben.

In krassem Gegensatz zur schulischen Realität der Kinder stehen die hohen Bildungserwartungen der türkischen Eltern; ${ }^{15}$ die Kinder sollen die Chancen, die das deutsche Bildungssystem bietet, nutzen und auch akademische Abschlüsse erreichen. Die klassischen Berufsziele sind Arzt, Ingenieur und Rechtsanwalt. Aus der Sicht der Stadtteilschulen jedoch sind das unrealistische Vorstellungen, da die meisten Eltern nur geringe Schulerfahrung haben ${ }^{16}$ und nicht wüssten, wie sie die Kinder fördern sollten. ${ }^{17}$

\section{Typische Entwicklungskarrieren türkischer Migrantenkinder im "Ghetto"}

Im Kindergarten- und Grundschulalter definieren sich die Kinder ethnisch, d.h. sie verstehen sich im Kontrast zu Kindern anderer Ethnien als „Türke", „Italiener", „Kroate" oder „Iraker". Die Selbstdefinition steht dabei in enger Relation zur Familiensprache; „Türkisch" sein bedeutet „Türkisch" sprechen. Mit dem Verlassen der Grundschule bilden die Kinder zwei grundsätzlich verschiedene soziale und berufliche Orientierungen aus, die zu unterschiedlichen Selbstbildern und unterschiedlichen Sprach- und Kommunikationspraktiken führen:

$10 \mathrm{im}$ Ghetto bleiben und sich damit identifizieren und 1 laus dem Ghetto streben und sich davon distanzieren.

$\mathrm{Zu}$ a) Die Herausbildung dieser Orientierung hängt sehr eng mit dem Übergang zur Hauptschule zusammen. Da die Hauptschule auf dem Territorium des Ghettos liegt, bedeutet die Hauptschulempfehlung nach der 4.Klasse, dass die weitere Entwicklung des Kindes im Lebensraum des Ghettos verläuft. ${ }^{18}$ Da den meisten Hauptschülern der schulische Erfolg versagt ist, entwickeln sie bereits sehr früh (oft schon mit 11-12 Jahren) eine ,anti-schulische" und ,anti-berufliche" Haltung und suchen außerhalb der Schule nach neuen Herausforderungen und nach Anerkennung, die sie z.B. in ethnischen Cliquen $^{19}$ oder in religiösen und politischen Gruppen finden. ${ }^{20}$ Das Selbstbild dieser Jugendlichen

15

Unsere Erfahrungen - und auch die Auskünfte der Lehrenden weisen in dieselbe Richtung widerspre

chen den Feststellungen, dass türkische Eltern wenig Interesse an der Bildung ihrer Kinder hätten. Die von uns befragten Eltem sorgen sich sehr, dass ihre Kinder in der Schule nicht vorankommen, und sie sind ratlos, wie sie ihren Kindern helfen können.

16 Nach Auskunft der Schulen geht das aus Schulerhebungen und aus Berichten der Kinder

hervor.

17 Nach Meinung einer deutschen Lehrerin wissen die Eltern nicht, was lernen heißt. Das bestätigt türkischer Kollege: Die meisten Eltern kommen aus anatolischen Bergdörferm und sie wissen nicht, wie man lernt und schon gar nicht 8-9 Jahre lang.

18 Im Ghetto gibt es weder ein Gymnasium noch eine Realschule. Außerdem erfuhr ich von Pädagoginnen,

dass, um den Bestand an Hauptschulen und Lehrdeputaten zu erhalten, vor allem Migrantenkinder die Hauptschulempfehlung bekämen, und dass damit die Existenz der Hauptschulen gesichert würde.

19 Vgl. dazu meine Beschreibung der Entwicklung der „Powergirls", Keim in Vorb., Teil II

20 Dazu haben wir keine eigenen Untersuchungen gemacht. Vor der Gefahr, dass türkische Jugendliche,

die im deutschen Schulsystem scheitern und sich von der Mehrheitsgesellschaft abgelehnt fühlen, sich in religiös- und politisch-fundamentalistischen Organisationen engagieren, um dort Anerkennung zu finden und ein positives Selbstwertgefühl aufzubauen, haben Heitmeyer/Müller/Schröder in ihrer Unter suchung „Verlockender Fundamentalismus" bereits 1997 gewarnt. Nach ihren Erkenntnissen lassen sich „Jugendliche, die sich aufgrund alltäglicher Unterlegenheitserfahrungen nach demonstrativer Stärke 
ist typischerweise ethnisch geprägt und ihre sozialen Netzwerke sind auf die Migrantengemeinschaft begrenzt.

Da der Bildungsdruck in türkischen Familien oft sehr hoch ist, ist für die Kinder mit der Hauptschulempfehlung ein Imageverlust verbunden. Eine Hauptschülerin beschreibt, dass sie in der Familie nur die Blöde war, die nichts versteht, die es nicht geschafft hat, während die Geschwister, die höhere Schulen besuchten, bewundert wurden. Solche Erfahrungen fördern die Ausprägung ,anti-schulischer" Haltungen und treiben die Betroffenen in Aktivitäten, mit denen sie ein positives Selbstbild entwickeln können. Nach dem Verlassen der Hauptschule setzt sich die Begrenzung des Lebens der Jugendlichen auf das Ghetto fort: Sie bleiben mit ihren Freundinnen zusammen und suchen Anlernoder Aushilfsjobs im Ghettokontext, da sie - so ein türkischer Pädagoge - Angst vor einer neuen Umgebung haben, in der sie sich alleine zurechtfinden müssten. In einer Jugendgruppe z.B. hat einer der Jungen eine Lehrstelle außerhalb Mannheims gefunden. Seine Freunde halten das für abartig, und der Junge ist - so sein Lehrer - eine absolute Ausnahme.

Die räumliche und soziale Geschlossenheit des Ghettos ist auch der Lebensraum vieler junger Erwachsener: Sie heiraten entweder Partner aus ihrem türkischstämmigen Umfeld oder aus den Herkunftsregionen ihrer Familien in der Türkei, und wohnen dann bei den Eltern oder suchen Wohnraum im Umkreis ihrer Familien im Ghetto. ${ }^{21}$

Im Familienkontext sprechen diese Ghettojugendlichen Türkisch oder „Migrantentürkisch", eine Form des Türkischen, die sich in der Migration herausgebildet hat; ${ }^{22}$ mit gleichaltrigen Türkischstämmigen werden oft deutsch-türkische Mischungen mit mehr oder weniger hohen türkischen Anteilen verwendet; und in der multilingualen Peergroup, im Klassenverband, in Sport- oder Musikgruppen ist „Ghettodeutsch" die Normalform, eine ethnolektale Form Deutschen mit ganz bestimmten Merkmalen (vgl. unten, Kap. 4.2). Wenn die jungen Leute keinen Aktivitäten außerhalb der Migrantengemeinschaft nachgehen, spielt Umgangsdeutsch in ihrem Leben kaum eine Rolle.

$\mathrm{Zu}$ b) Die Empfehlung für eine höhere Schule bedeutet, dass die Kinder mit der 5. Klasse aus dem Ghettoumfeld in eine neue schulische und soziale Welt eintreten, die deutsch dominiert ist und in der sie eine Minderheit bilden. Mit diesem Übergang, den die meisten Kinder ersehnen, aber nur wenige schaffen, sind ganz andere Probleme verbunden. In der Welt außerhalb des Ghettos werden sie mit sprachlichen, schulischen und sozialen Anforderungen konfrontiert, bei deren Bewältigung ihnen in der Regel niemand hilft. Versagens- und Fremdheitserfahrungen können ernste Identitätskrisen auslösen und bis

\footnotetext{
sehnen, leicht von Organisationen, die Macht beanspruchen oder ausstrahlen, für ihre Zwecke benutzen. (...) Aus einem möglicherweise nur (jugend)kulturellen Problem wird somit ein politisches"; zitiert aus der „Zeit", Nr. 35, 1996.

Frau Die enge, ganz auf die türkische Großfamilie begrenzte Lebenswelt junger Paare, in der die

ratsmigrantin ist, beschreibt die Soziologin N. Kelek sehr einprägsam in ihrem Bericht Die fremde Braut. Bericht aus dem Inneren des türkischen Lebens in Deutschland", der auf eigenen Erfahrungen und Gesprächen mit 50 Heiratsmigrantinnen basiert.

22 Zur Beschreibung des Migrantentürkisch in Mannheim vgl. Cindark/Aslan 2004 und Sirim 2004.
}

21 
zum schulischen Scheitern führen. In dieser Phase brauchen die Kinder schulische Unterstützung und positive Leitbilder, die ihnen die neue Welt erklären helfen und ihnen zeigen, wie sie den schwierigen schulischen und sozialen Aufstieg meistern können. Gelingt ihnen dieser Weg, erwerben sie in der Regel eine hohe Kompetenz in Deutsch (schriftlich und mündlich) und sie entwickeln ein Selbstbild, das im Kontrast zu dem der Ghettojugendlichen steht: Sie orientieren sich an Leitbildern jenseits enger ethnischer Grenzen, distanzieren sich von den traditionellen sozialen Orientierungen der Ghettogemeinschaft und sind auf den beruflichen und sozialen Erfolg hin - national oder international - orientiert. ${ }^{24}$ Für eine der Gruppen, die „Powergirls", ist ein elaboriertes deutschtürkisches Mixing Symbol für ein eigenständiges, bilinguales und bikulturelles Selbstverständnis (vgl. unten Kap. 5.2).

\section{Sprach- und Kommunikationspraktiken der Migrantenkinder und -jugend- lichen im Ghetto}

\subsection{Die Herausbildung von Mixing-Praktiken im Kindergarten ${ }^{25}$}

Da die Kindergärten des Stadtgebiets einen hohen Migrantenanteil haben (bis zu 100 \%), lernen die Kinder im Kindergarten nur wenig Deutsch. Sie spielen entweder in der nationalen Gruppe und sprechen die Herkunftssprache gemischt mit deutschen Bezeichnungen, die sie von den Erzieherinnen übernehmen. Oder sie spielen mit Kindern anderer Herkunftssprache; die Verkehrssprache ist dann eine frühe Lernervarietät des Deutschen gemischt mit Wörtern und Strukturen aus anderen Sprachen. Kinder, die einen Großteil der Zeit in solchen Spielkonstellationen verbringen, bilden eine eigene Kommunikationsform aus, die für Außenstehende oft schwer verständlich ist. Ihnen fehlen Sprachvorbilder für Deutsch, da im normalen Kindergartenalltag der sprachliche Einfluss der deutschsprachigen Erzieherinnen eher gering ist. ${ }^{26}$

Solche biographischen Erfahrungen machten fast alle „Powergirls"; einige verließen das Gymnasium

vorzeitig und schafften erst später, als sie in stabiler waren, den Weg zum Abitur, vgl. Keim i. Vorb., Teil II. Es gibt auch einen prominenten Vertreter für das schulische Scheitern außerhalb des Ghettos: Der Berliner Hip-Hoper Fuat, der aus dem Ghetto kommt und den Weg ins Gymnasium außerhalb des Ghettos zunächst geschafft hat, beschreibt, dass er dort ,fertig gemacht" wurde und die Schule vorzeitig verließ.

Alle drei von uns untersuchten Migrantengruppen, die „Powergirls", die „Europatürken" und die ,Un mündigen" haben diesen Weg geschafft. Jede Gruppe hat im Vergleich zur Ghettopopulation eine neue sozial-kulturelle Orientierung entwickelt und im Zusammenhang damit eigenständige Sprach- und Kommunikationspraktiken. Zu einem Vergleich der drei Gruppen, ihrem unterschiedlichen Selbstver ständnis und ihren unterschiedlichen Sprach- und Kommunikationspraktiken vgl. Keim 2003. Zur Gruppe der „Europatürken" vgl. Aslan (in diesem Band) und zur Gruppe der „Unmündigen" vgl. Cindark (in diesem Band).

25 Das Folgende wurde von $\mathrm{S}$. Aslan und $\mathrm{mir}$ in mehreren Vorträgen und Fortbildungsveranstaltungen

vorgestellt. Die Gesprächsbeispiele aus dem Kindergarten stammen aus dem Korpus, das S. Aslan er stellt hat.

Ähnlich wie die Lehrkräfte in den Schulen sind auch die Erzieherinnen auf die Anforderungen in multi lingualen Ghetto-Kindergärten nicht vorbereitet. Erst in den letzten Jahren werden sie in Fortbildungs veranstaltungen auf die besonderen Aufgaben der Sprachförderung von Migrantenkindern vorbereitet. 
Interessant sind vor allem die Mixing-Praktiken in türkischstämmigen Kindergruppen. ${ }^{27}$ Wenn die Kinder mit 3-4 Jahren in den Kindergarten kommen, haben sie in der Familiensprache bereits eine hohe Sprachkompetenz entwickelt, vor allem wenn ein Elternteil Heiratsmigrant/in ist. In der türkischstämmigen Spielgruppe entwickeln sich dann türkisch-deutsche Mischungen. Die Kinder übernehmen Bezeichnungen, die sie von den Erzieherinnen gehört haben, und betten sie in ihre türkischen Konstruktionen ein, wie die folgenden Beispiele zeigen:

a) In dem Ausschnitt erinnert ein türkischer Junge seinen Freund daran, dass beide nach den Ferien in die Schule kommen: ${ }^{28}$

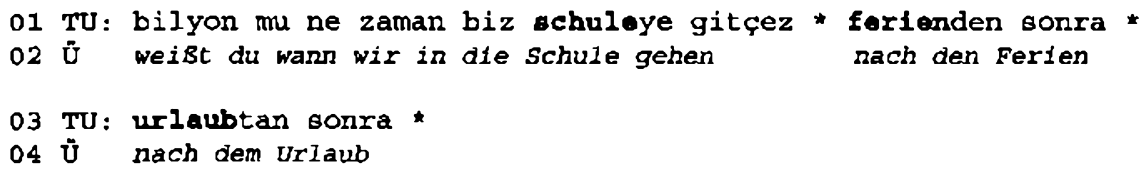

In die türkische Äußerung sind die deutschen Nomina Schule, Ferien und Urlaub eingebettet und mit türkischen Kasus-Morphemen verbunden: Schule ist mit dem Dativmorphem -ye und Ferien und Urlaub sind mit den Ablativmorphemen -den bzw. -dan verbunden, die nach den Regeln der türkischen Vokalharmonie verändert werden.

b) Im nächsten Beispiel berichtet der 6jährige Onur seinen Freunden, welche Anweisung ihm die Erzieherin gegeben hat:

OI ON: dedi schrank/ schranka legen dedi

02 Uั sie sagte Schrank/ in den Schrank legen sagte sie

Die Anweisung der Erzieherin (in den) Schrank legen ist in die türkische Struktur dedi (sie sagte) eingebettet und mit dem Dativ-Morphem - $a$, der türkischen Morphologie entsprechend, verbunden: schranka legen dedi (in den Schrank legen sagte sie).

Interessant ist, dass die Kinder für die Welt im Kindergarten deutsche Bezeichnungen verwenden, während sie für die türkische Lebenswelt entsprechende türkische Bezeichnungen gebrauchen. D.h. mit dem Eintritt in den Kindergarten wird das Lexikon lebensweltspezifisch ausdifferenziert. Wenn die Kinder z.B. über den Kräutertee, den sie im Kindergarten bekommen, sprechen, verwenden sie den Ausdruck tee trinken, wie in der Äußerung ben tee trinken etcem (wörtlich: ich will Tee trinken machen). Wenn sie zuhause (schwarzen) Tee trinken, wird das mit çay içmek (Tee trinken) bezeichnet.

Auch für Beziehungen zu deutschen Personen aus dem Kindergarten entwickeln die Kinder neue Bezeichnungen. Im folgenden Beispiel erzählt der 6jährige Ömür der Interviewerin, dass er und sein Freund für die neue Praktikantin, Frau Hanke, schwärmen:

01 OM: çunkŭ frau Hankeyi biz aeviyoz

02 Uิ weil wir Frau Hanke lieben

$03 \mathrm{SE}$

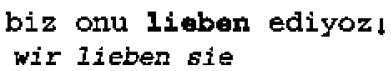

27 Mixing in türkischstämmigen Kinder- und Jugendgruppen wurden in Deutschland auch von

Dirim

1998

Dirim/Auer 2004, Hinnenkamp 2005 und Pfaff 1999 beschrieben; in Holland von Ad Backus 1996 und in Dänemark von Norman Jorgensen 1998.

28 Die deutschen Bezeichnungen sind fett. 
In der ersten Äußerung bezeichnet Ömür das Gefühl, das er und sein Freund Frau Hanke gegenüber empfinden durch das türkische Verb seviyoz (wir lieben/ mögen sie) in çünkü frau Hankeyi biz seviyoz $\downarrow$. Dann reformuliert er die Äußerung und präzisiert zu biz onu lieben ediyoz (wörtlich: wir tun sie lieben). Er verwendet das deutsche Verb lieben, verbindet es mit dem türkischen Verb etmek (machen, tun) und kreiert zum Ausdruck seines besonderen Gefühls zu Frau Hanke die neue gemischtsprachliche Verbform lieben ediy$o z$. Wenn er dagegen von seinem Gefühl der Mutter gegenüber spricht, verwendet er den türkischen Ausdruck onu seviyorum (ich liebe sie).

Wie diese Beispiele zeigen, entstehen die deutsch-türkischen Mischungen primär nicht aus Gründen der Wortsuche, d.h. weil ein bestimmtes Wort in der einen Sprache nicht präsent ist und kurzfristig in die andere Sprache gewechselt wird. Die natürliche Voraussetzung für die Herausbildung sprachlicher Mischungen scheint die stabile Teilhabe der Kinder an zwei unterschiedlichen sozialen und sprachlichen Welten zu sein: die Welt der türkischsprachigen Kindergruppe, die in die Welt des deutschen Kindergartens mit neuen deutschen Bezugspersonen eingebettet ist. Die Kinder differenzieren ihren Wortschatz den Anforderungen in diesen unterschiedlichen Welten entsprechend aus. Wie Nachprüfungen ergeben haben, ${ }^{29}$ beherrschen die Kinder auch oft die türkischen Bezeichnungen für Objekte, die in den Mischungen in Deutsch bezeichnet werden. Die deutschen Bezeichnungen haben für sie jedoch eine andere Konnotation.

Es gibt natürlich auch Einbettungen deutscher Wörter in die türkische Struktur, die auf Wortsuche hindeuten; ${ }^{30}$ in diesen Fällen wird der Wechsel ins Deutsche durch Pause, Verzögerungspartikel oder Proformen wie Dings oder şey (Ding) markiert, wie im folgenden Beispiel:

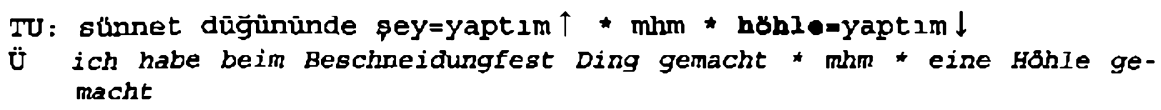

In der Äußerung ist die Wortsuche durch die Profom şey (Ding), Pause und Verzögerungspartikel $\mathrm{mhm}$ markiert. Danach erfolgt der Wechsel ins Deutsche und die passende, deutsche Bezeichnung Höhle wird in die türkische Struktur eingebettet höhle=yaptim $\downarrow$ (ich habe eine Höhle gemacht).

Die bisher beschriebenen Mixingstrukturen werden von den Kindern entwickelt, die in der Familie ausschließlich Türkisch sprechen, weil z.B. ein Elternteil Heiratsmigrant/in ist oder sie von den Großeltern betreut werden. Ist die Mutter bzw. Bezugsperson in Deutschland sozialisiert und wird in der Kommunikation mit dem Kind auch Mixing verwendet, produzieren solche Kinder Mixingstrukturen, wie wir sie für die Jugendlichen beschrieben haben (vgl. unten, Kap. 5.2). Diese Mixingstrukturen sind komplexer, Sprachwechsel finden auch an syntaktischen Grenzen statt und sie haben diskursive Funktionen. Das möchte ich am Beispiel eines 6 Jährigen zeigen, dessen Mutter im Stadtgebiet aufgewachsen ist und die mit dem Jungen häufig gemischt spricht. Im Transkriptausschnitt erzählt der Junge der bilingualen Interviewerin, dass er im Kindergarten aufgrund seiner Sprachkompetenzen eine Sonderrolle einnimmt:

29 Vgl. S. Aslan i. Vorb.

30 In der Terminologie von Poplack sog. „flagged switches" (1980, S. 
$01 \mathrm{TU}:$ waihnachtomann geln/gel/ gelmişti $\uparrow$ isch hab zwel cukuladen
$02 \mathrm{U}$ der weihanchtamann gekom/ war gekommen

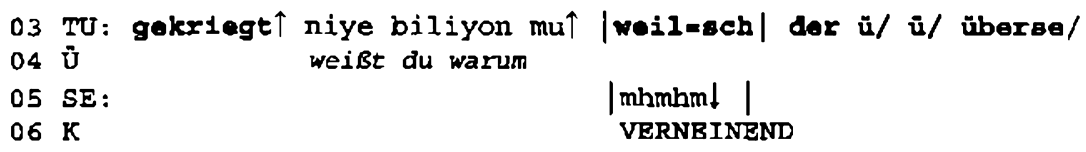

[...]

16 TU: zaten isch helf die alle $\uparrow$ un=dann krigg lach alles

17 Uั sowieso

18 TU: póh $\downarrow$ cocuk/ çocuklar $\uparrow$ bauste1neleri yapmıyolar $\uparrow$ ya dökmüsler $\uparrow$

19 die kind/ die kinder machen die bausteine nicht also haben sie

$20 \mathrm{TU}$ : yapmiyolar $\uparrow$ * be ${ }^{\mathrm{n}} \mathrm{n}$ yapıyon $\downarrow$ krlgg i/ krigg 1ach lutbcher $\downarrow$

21 Ü verstreut sie machen (das) nicht *ich mach das *

In dieser Erzählung haben einige Sprachwechsel diskursive Funktion und dienen der Konturienung zweigliederiger Darstellungsstrukturen und der Unterscheidung zwischen:

a) Hintergrund und Vordergrund: Eine solche Struktur produziert der Junge gleich zu Beginn seiner Erzählung:

01 TU weibnachtbmann gel/gelmigti $\uparrow$ iech hab zwei cukuladen gekriegt $\downarrow$

Der erste Äußerungsteil, der in die Szene einführt Weihnachtsmann gel/gelmişti (der Weihnachtsmann war gekommen), hat eine türkische Struktur, in die das deutsche Nomen Weihnachtsmann, eine kindergartenspezifische Bezeichnung, ${ }^{31}$ eingebettet ist. Der zweite Äußerungsteil, in dem der Junge das für ihn Besondere schildert, ist in Deutsch formuliert: isch hob zwei çukuladen gekriegt. Das Nomen çukuladen ist eine Überblendung aus dem türkischen çikolata und dem deutschen Schokolade. Die Äußerung hat folgende Sprachwechselstruktur:

Hintergrund: Türkisch

Weihnachtsmann gelgelmişti
- Vordergrund: Deutsch

isch hab zwei çukuladen gekriegt

31 Weihnachtsmann ist im engen Sinne kein cultural borrowing; es gibt im Türkischen eine entsprechende Bezeichnung noel baba. Die ist in Deutschland jedoch nur selten zu hören, so dass für die Kinder die einzig verfügbare Bezeichnung für den ,Weihnachtsmann' die deutsche ist; zu cultural borrowings und core borrowings, vgl. Balci (in diesem Heft). 
b) rhetorische Frage und Antwort: Diese Struktur erfolgt direkt im Anschluss:

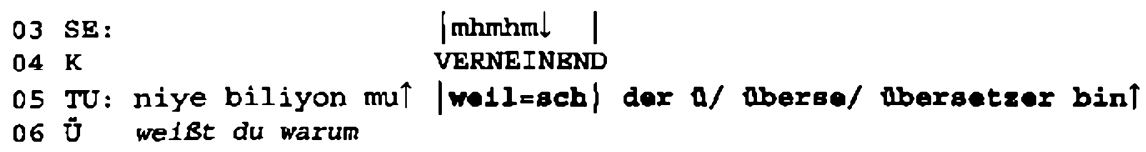

Die Frage niye biliyon mu^ (weißt du warum, 05), die dem Spannungsaufbau und der Vorbereitung der zentralen Information dient, ist in Türkisch. In der Antwort nennt der Junge dann den Grund für das Schokoladengeschenk und hebt seine eigene Leistung hervor; die Antwort ist in Deutsch: weil-sch der ü/ überse/Übersetzer bin $\downarrow$. Die gesamte Äußerung hat die Sprachwechselstruktur:

$\begin{aligned} & \text { Rhetorische Frage: Türkisch } \\ & \text { niye biliyon } m u \uparrow\end{aligned} \quad \begin{aligned} & \text { Antwort: Deutsch } \\ & \text { weil=sch der Übersetzer bin }\end{aligned}$

In der generalisierenden Feststellung zaten * isch helf die alle $\downarrow$ un=dann krigg isch alles $\downarrow$ (16) bringt der Junge seine besondere Rolle im Kindergarten auf den Punkt. Diese Äußerung ist bis auf den einleitenden Diskursmarker zaten (sowieso) in Deutsch formuliert. Danach folgt eine Detaillierung, in der der Junge schildert, dass die anderen Kinder ihre Bausteine nicht aufräumen. Dieses Formulierungssegment hat eine türkische Struktur, in die das deutsche Nomen bausteine insertiert und mit den türkischen Morphemen für Plural -ler und Akkusativ $-i$ verbunden ist. Dann folgt wieder eine zweigliedrige Struktur, in der durch Sprachwechsel unterschieden wird zwischen

c) Voraussetzung und Folge:

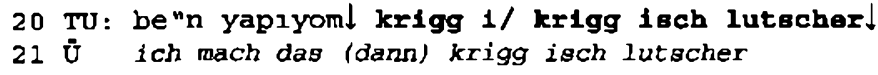

Im ersten Äußerungsteil beschreibt der Junge, dass er für die anderen Kinder aufräumt. Als Belohnung dafür bekommt er dann von der Erzieherin einen Lutscher. Dieser zweite Äußerungsteil, inhaltlich als Folge des ersten, ist mit einem Wechsel ins Deutsche verbunden. Die Äußerung hat dieselbe Sprachwechselstruktur wie die vorher dargestellten:

$$
\begin{aligned}
& \text { Voraussetzung: Türkisch } \quad-\quad \begin{array}{c}
\text { Folge: Deutsch } \\
\text { be " } n \text { yapiyom } \downarrow
\end{array} \\
& \text { krigg isch lutscher }
\end{aligned}
$$

Die Beispiele zeigen, dass die von dem Jungen verwendeten Sprachwechsel der Strukturierung der Darstellung dienen; durch die Wechsel werden einzelne Strukturteile zusätzlich konturiert und fokussiert. Dabei ist die Sprachenfolge geregelt: der erste Strukturteil ist in Türkisch, der zweite, hervorgehobene Teil in Deutsch. Der Junge hat diese Mixingstrukturen, die ähnlich wie die Strukturen sind, die wir bei den „Powergirls" feststellten, von seiner Mutter und deren Familie gelernt. Da diese Familie und die Gruppe der "Powergirls" keinen Kontakt miteinander haben, deutet das darauf hin, dass solche Mixingstrukturen im Ghetto unter Angehörigen der zweiten und dritten Migrantengeneration verbreitet und relativ stabil sind.

Interessant ist außerdem, dass die deutschen Segmente im Mixing des Jungen strukturell weit entwickelt sind; er beherrscht bereits große Bereiche der Grundstrukturen des Deutschen (Verbflexion, Subjekt-Verb-Kongruenz, Inversionsregel, Haupt- und Nebensatzstruktur, verschiedene Tempora). Im Vergleich dazu ist das Deutsch der gleichaltri- 
gen türkischstämmigen Kinder, deren Mütter Heiratsmigrantinnen sind, noch nicht so weit entwickelt. Das zeigt das folgende Beispiel, in dem ein 6jähriges Mädchen versucht, der Interviewerin das Märchen „Schneewittchen" in Deutsch zu erzählen:

01 FE: wir/ wir/ die und ich * böse kind $\downarrow$ und die schönes kind!

02 FE: un die kuck ăh bulume t un bulume u/ uns gehört $\uparrow$ und wir/

03 FE: die gebt die apfel $\uparrow$ damit die beist $\downarrow$ un da die=s tot $\downarrow$

Die Sätze sind kurz und bestehen aus wenigen Elementen. Die ersten Äußerungssegmente sind dem Märchen „Schneewittchen" nicht zuordenbar, und nur der letzte Äußerungsteil (03) enthält Strukturelemente des Märchens. D.h., das Kind kann die Geschichte, die die Erzieherin erzählte, nicht richtig wiedergeben.

\subsection{Die Herausbildung von "Ghettodeutsch" in der Schule}

Mit „Ghettodeutsch" bezeichne ich die Form des Deutschen, die sich in dem Stadtgebiet unter Migrantenkindern unterschiedlicher Herkunft als eine Art Ethnolekt herausgebildet hat. ${ }^{32}$ Sprachformen, die dem Ghettodeutsch in Mannheim ähnlich sind, haben sich auch in anderen Städten Deutschlands herausgebildet. Füglein (1999) beschreibt sie für Bamberg und München als ethnolektale Varietät des Deutschen, als „Kanak Sprak", ${ }^{33}$ und stellt folgende Charakteristika fest: Verberststellung und Ausfall verschiedener Elemente wie Präpositionen, Artikel, der Proformen „es" und „des" in Nominativ- und Akkusativposition. Aus dem Türkischen stammen Adressierungen, Droh- und Schimpfformeln. ${ }^{34}$ Auffallend seien prosodische und phonetische Eigenschaften, die die Autorin als „stakkatoartiges" Sprechen mit „fremdländischem Akzent" bezeichnet. „Kanaksprachliche" Elemente sind nach Beobachtung der Autorin sozial markiert und werden vor allem von Jugendlichen mit niedrigem Bildungsgrad verwendet. Auch Dirim/Auer (2004) stellen unter Hamburger Jugendlichen ethnolektale Formen mit ähnlichen Merkmalen fest, vor allem im prosodischen und phonetischen Bereich (z.B. Koronalisierung von [c] zu [sch], gerolltes [r] auch in Anlautclustern, der silbenzählende Rhythmus), aber auch im morphosyntaktischen Bereich (Wegfall von Präpositionen und Artikel, ungewöhnliche Verwendung von Adverbien und Präpositionen, unübliche Verbspitzenstellung). ${ }^{35}$ Nach Beobachtung der Autoren werden solche Formen sowohl von Migrantenjugendlichen, als auch von deutschen Jugendlichen im Gespräch mit Migranten verwendet. ${ }^{36}$

Die Bezeichnung „Ghettodeutsch" übemehme ich von einem türkischstämmigen Informanten, der diese

Sprachform als „Deutsch dieser Ghettomenschen" bezeichnet und ihren Symbolwert folgendermaßen beschreibt: „So spricht der typische Migrantenjugendliche, der sich als Macho darstellen will, als cool, aggressiv und Sex bezogen (...). Ein gebildeter Türke spricht so nicht Deutsch".

33 Füglein 1999 übernimmt die Bezeichnung, die der Schriftsteller Feridun Zaimoğlu (1995) mit seinem gleichnamigen Buch einführte.

34 z.B. Adressierungen wie hey lan (=he Mann) oder Formeln wie hadi lan (=los Mann), siktir (=verpiss dich Mann) oder inek (=kuh)

35 Vgl. Dirim/Auer 2004, Kap. 6.3.

36 Auer (2003) bezeichnet ethnolektale Formen des Deutschen, die von türkischstämmigen Jugendlichen

gesprochen werden, als „Türkenslang". Er unterscheidet zwischen einem primären Ethnolekt, der in na türlichen Jugendgruppen (auf der Straße, in der Schule) gesprochen wird, einem sekundären Ethnolekt, den medialen Verarbeitungen und Stilisienungen des primären Ethnolekts, und einem tertiären Ethno lekt, den ethnolektalen Formen, die deutsche Jugendliche zur Abgrenzung oder zur Karikatur von typi schen Primärsprechem verwenden. Wenn in typischen Migrantenwohngebieten auch nicht-türkische 
Eine Sprachform, die strukturelle Ähnlichkeiten mit den genannten ethnolektalen Deutschvarietäten hat, hat sich unter Migrantenjugendlichen in den Vororten Stockholms herausgebildet. Kotsinas (1998) beschreibt das "Rinkeby-Schwedisch" oder die „Kebabsprak" als eine Sprachform, die von Jugendlichen unterschiedlicher Herkunft (Türkisch, Griechisch, Chinesisch, Chilenisch und Schwedisch) gesprochen wird, und von Stockholmer Varietäten abweicht. ${ }^{37}$ Während einige Jugendliche vorwiegend Rinkeby-Schwedisch sprechen, wechseln andere je nach situativen Anforderungen zwischen Schwedisch und Rinkeby-Schwedisch. Rinkeby-Schwedisch dient, so die Autorin, den Jugendlichen als Mittel zum Ausdruck einer eigenen Identität im multiethnischen Migrationskontext.

Das „Ghettodeutsch", das sich in Mannheim herausgebildet hat, hat strukturelle Ähnlichkeiten mit den genannten ethnolektalen Varietäten. Es ist eine vereinfachte Form der deutschen Umgangssprache, in der einige Charakteristika relativ durchgängig, andere auch nur vereinzelt vorkommen:

Relativ durchgängig kommen vor:

- Wegfall von Präposition und Artikel in Lokal- und Richtungsangaben: z.B. isch muss Toilette, isch geh Schule

- Ausfall des Artikels in Nominalphrasen: z.B. gib mir Kippe

- Generalisierung einiger Verben gehen, kommen und vor allem machen: z.B. isch mach disch Krankenhaus (,ich schlag dich krankenhausreif.")

- Verwendung von Formeln wie isch schwör zur Bestätigung und isch hass des zur negativen Bewertung

- Verwendung türkischer Formeln zur Anrede (lan, moruk, „Mann", „Alter"), zur Beschimpfung (siktir lan ,verpiss dich") und als Interjektionen und Diskursmar-ker

- eine spezielle Art der Informationsvermittlung, die einen hohen Grad an geteiltem

Wissen voraussetzt und nur einen geringen Teil dessen explizit macht, was für den Gesprächspartner zum Verständnis notwendig ist ${ }^{38}$

Form

ein Komplex phonologisch-prosodischer Merkmale, der zu einer besonderen

des Sprechens führt, dem ,gestoßenen" Sprechen (vgl. dazu unten, S. 214).

Jugendliche solche Formen sprechen, spricht Auer von „Deethnisierung" des Ethnolekts, d.h. er wird zur normalen Umgangssprache zwischen Jugendlichen unterschiedlicher Herkunft, auch deutschen Jugendlichen. Die von mir verwendete Bezeichnung „Ghettodeutsch" umfasst das, was Auer als deethnisiertes Türkendeutsch bezeichnet. Meiner Beobachtung nach bildet und stabilisiert sich das Ghettodeutsch in multilingualen Gemeinschaften, in denen die Türkischstämmigen eine große Rolle spielen.

Abweichungen betreffen vor allem Intonation und Rhythmisienung, gerolltes /r/, Einebnung der Unter scheidung zwischen kurzen und langen Vokalen, grammatische Vereinfachungen (z.B. Bevorzugung nur einer Genusform und nur weniger Präpositionen, analytische Tempusbildung), abweichende Wort stellung (Inversion) und die Übemahme von Anredeformen, von Slang- und Schimpfwörtem aus ver schiedenen Sprachen.

38 Vgl. die Analyse in Kallmeyer/Keim 2003a. 
Gelegentlich kommen vor:

- Ausfall von Pronomina und suppletiven Elementen: z.B. wann has $=d u$ (sie) fotografiert

- Andere Genera: z.B. rischigtes Tee

- Andere Verbrektion: $\mathrm{z}: \mathrm{B}$. wenn $=$ sch mit ihm heirate

- Andere Wortstellung: z.B. Hauptsache lieb isch ihn

Als Beispiel für Ghettodeutsch ein kurzer Ausschnitt aus einem Gespräch zwischen 17jährigen Ghettojugendlichen aus einer Berufsvorbereitungsklasse. ${ }^{39}$ Die türkischstämmigen Jungen Onur (ON) und Can (CA) sprechen mit dem arabischen Rafi (RA) über eine Gruppe Jugendlicher aus dem Jugendzentrum „Erlenhof", mit denen sie im Streit liegen und die voll die Fische sind (Idioten):

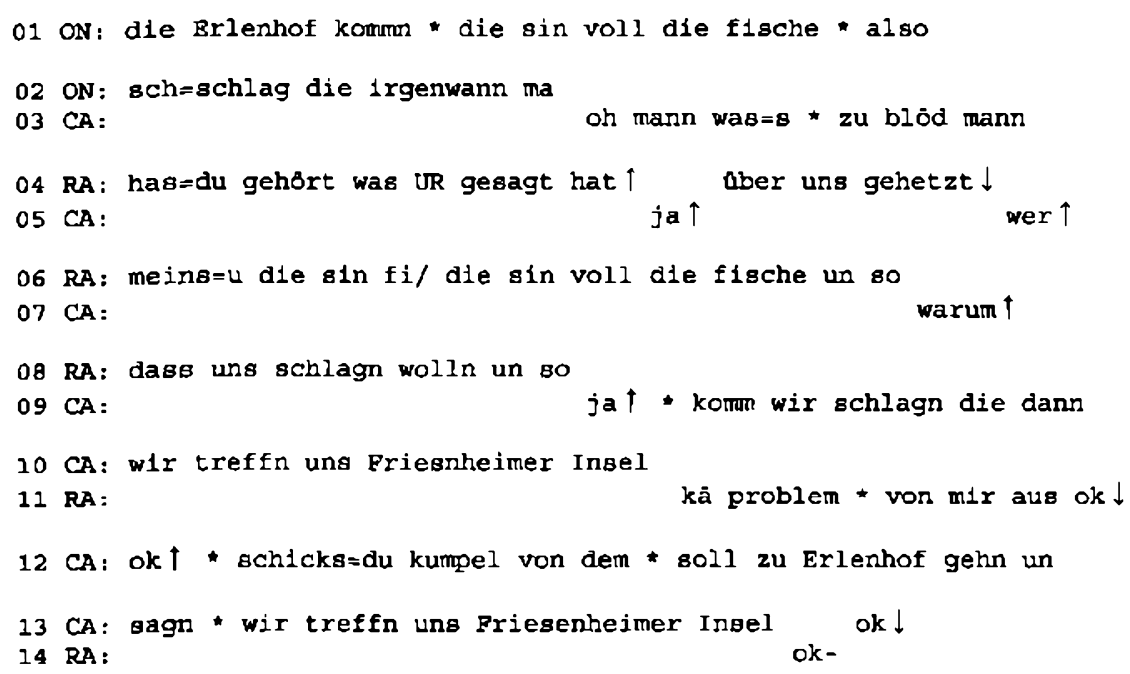

Ghettosprachliche Merkmale sind der Ausfall von Artikel und Präposition in Präpositionalphrasen, wie die Erlenhof kommen (die vom Erlenhof kommen) oder wir treffen uns Friesenheimer Insel (wir treffen uns auf der Friesenheimer Insel), Ausfall des Artikels in schicks-du kumpel von dem (schickst du einen Kumpel von dem) und in zu Erlenhof (zum Erlenhof); Ausfall des Personalpronomens in soll zu Erlenhof gehen (er soll zum Erlenhof gehen).

Zu Fragen der Stabilität des Ghettodeutsch und vor allem zu Fragen des Repertoires der jugendlichen Sprecher kann ich z.Zt nur einige Beobachtungen anführen. Die Jugendlichen aus dem Berufsvorbereitungsjahr, von denen das Gesprächsbeispiel stammt, verwenden kaum Umgangsdeutsch; auch Betreuenden und Erziehenden gegenüber kommt

39 Die Klassen im Berufsvorbereitungsjahr (BVJ) bestehen zu über $90 \%$ aus Migrantenjugendlichen. Die meisten haben keinen oder einen schlechten Hauptschulabschluss und kaum eine Chance auf eine qualifizierte Berufsausbildung. Im BVJ haben sie die Möglichkeit den Hauptschulabschluss nachzumachen bzw. sich auf eine Anlemtätigkeit vorzubereiten. Nach Meinung von Lehrenden ebenso wie von Jugendlichen selbst ist das BVJ „ein Abstellgleis" und ein Sammelbecken für „loser" (Verlierer). 
vor allem Ghettodeutsch vor. D.h. es scheint Migrantenjugendliche zu geben, deren Sprach- und Kommunikationsverhalten keine situationelle Ausdifferenzierung aufweist und die, wenn sie Deutsch sprechen, bevorzugt ghettosprachliche Formen verwenden. Dagegen habe ich in einer der 8. Hauptschulklassen Folgendes beobachtet: Die Jugendlichen, auch deutsche Jugendliche, sprechen untereinander Ghettodeutsch. Doch sie verwenden grammatisch korrekte Formen und „Höflichkeitsregeln", wenn sie mit deutschen Lehrerinnen sprechen. Das zeigen die folgenden Beispiele: Ein 15jähriger kroatischer Schüler, der seinen Klassenfreund auffordert komm * wir gehen Pause, wendet sich kurze Zeit später an die Klassenlehrerin mit der Bitte: darf isch ma bitte auf die Toilette Frau Brand. Ein türkischstämmiger Schüler fragt seinen Betreuer, mit dem er sich freundschaftlich verbunden fühlt Güven abi * wann gehen wir Schwimmbad. Als er mit seinem Klassenlehrer spricht, verwendet er grammatisch korrekte Formen: isch hob die abber auf $=m$ Hof gesehn Herr Wolf $*$ die is nisch in der Klasse. ${ }^{40}$ Die situationeile Differenzierung zeigt, dass diese Jugendlichen Situationen, in denen Umgangsdeutsch erforderlich ist, von Situationen unterscheiden, in denen ghettosprachliche Formen die Normalform darstellen. D.h. ghettosprachliche Formen haben für sie gruppensprachliche Qualität. Wenn diese Jugendlichen ghettosprachliche Formen gegenüber deutschen Lehrerinnen verwenden, werden sie entweder korrigiert oder sie haben subversive und provokative Funktionen. ${ }^{41}$

Mit Beginn des Schullebens lernen Migrantenkinder zwischen Umgangsdeutsch, das die Lehrenden sprechen und Ghettodeutsch, das die Normalform in der multilingualen Klasse ist, zu unterscheiden. Die Kinder erleben, dass die Lehrenden ghettosprachliche Formen ablehnen und korrigieren, und sie lernen sehr schnell, dass sie die Lehrenden mit der Verwendung ghettosprachlicher Formen ärgern können. Das folgende Gespräch zwischen einem italienischstämmigen Erstklässler, Denis (DE), und seinem Lehrer (LE) stellt eine typische Lehrer-Schüler-Interaktion dar:

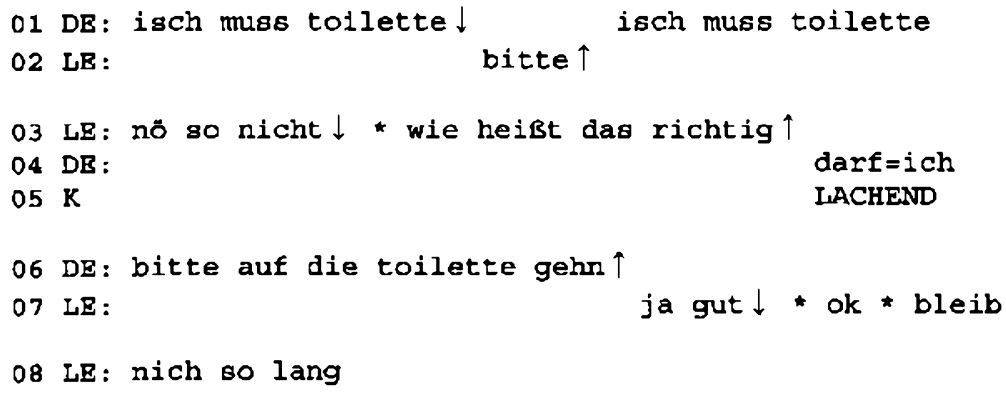

40 Vgl. dazu ausführlich Keim 2004. Neben Ghettodeutsch verwenden die untersuchten Jugendlichen auch

Sprachformen, die in den Medien als „Kanaksprak" oder als „Ausländerdeutsch" dargestellt werden, z.B. in Filmen von Erkan und Stephan und in CDs des Comedy-Duos „Mundstuhl". Das sind mediale Konstruktionen, die keine direkten Entsprechungen in der Realität haben. Dirim/Auer 2004 nennen solche Sprachformen „,sekundären Ethnolekt" (Kap. 6.13); vgl. auch die Darstellung in Androutsopoulos 2001 .

41 unterscheiden

Vgl. dazu die Analyse in Kallmeyer/Keim 2003; die dort untersuchten Jugendlichen tioneil sehr genau zwischen dem Gebrauch standardnaher Formen und ghettosprachlicher Formen und verwenden ghettosprachliche Formen zur Provokation der Lehrerin. 
Der Junge beginnt die Interaktion mit der Feststellung isch muss toilette $\downarrow$ (01). Die Äußerung hat Merkmale des Ghettodeutsch (Ausfall von Präposition und Artikel in der Richtungsangabe) und es fehlen Elemente konventioneller Höflichkeit. Der Lehrer reagiert mit einer Korrektur initiierenden Rückfrage bitte $\uparrow(02)$, mit der er dem Jungen anzeigt, dass mit seiner Äußerung etwas nicht stimmt, ihm aber gleichzeitig auch die Möglichkeit gibt, den Fehler selbst zu korrigieren. Doch der Junge ergreift die Korrekturgelegenheit nicht, er wechselt nicht in eine angemessene Anliegensformulierung, sondern wiederholt seine Eingangsformulierung und verstärkt sie damit. Jetzt reagiert der Lehrer explizit auf die Formulierung des Jungen nö so nicht $\downarrow *$ und fordert die richtige Form ein: wie heißt das richtig $\uparrow$ (03). Daraufhin kommt der Junge der Auffordenung nach und produziert, dabei leicht lachend, die angemessene und vom Lehrer erwartete Formulienung: darf ich bitte auf die toilette gehn (04/06). Diese Reaktion zeigt sehr deutlich, dass er die angemessene Formulierung kennt und weiß, was der Lehrer von ihm erwartet. Doch er genießt die Interaktion mit dem Lehrer, deren Verlauf für ihn vorhersehbar ist, und zeigt durch sein Lachen, dass es ihm Spaß macht, den Lehrer zu necken. Und der Lehrer kennt das Spiel und spielt es mit: er rügt den Jungen nicht, weil er auf der "falschen" Form beharrt, obwohl er die "richtige" kennt, sondern zeigt, dass er mit der Formulierung zufrieden ist, indem er ihm die Bitte gewährt.

Nach meiner bisherigen Beobachtung kommen ghettosprachliche Formen in der Ingroup-Kommunikation der Jugendlichen vor, solange sie sich im Ghettokontext bewegen. Das zeigt die Entwicklung der „Powergirls", die ich über mehrere Jahre verfolgen konnte. Solange die Mädchen in der Ghettohauptschule sind, treten in der Ingroup-Kommunikation Merkmale des Ghettodeutsch auf, die jedoch mit grammatisch korrekten Formen alternieren. Das zeigt das folgende Beispiel:

Im Transkriptausschnitt klären Teslime (TE), Tura (TU) und Hicran (HC), ob zwei weitere Mädchen in der Hauptschule oder der Realschule sind. Es wechseln deutschsprachige Redebeiträge mit türkischsprachigen, in die deutsche Lexeme insertiert sind. Dabei alternieren Lokalangaben ohne Präposition und Artikel mit grammatisch korrekten Lokalangaben. In den türkischen Äußerungen sind die Lokalangaben grammatisch korrekt realisiert. Bei den Schulbezeichnungen alternieren Vollformen mit Kurzformen: Hauptschule wird zu „Haupt" und Realschule zu „Real" verkürzt, und in dieser Form auch in die türkische Struktur insertiert:

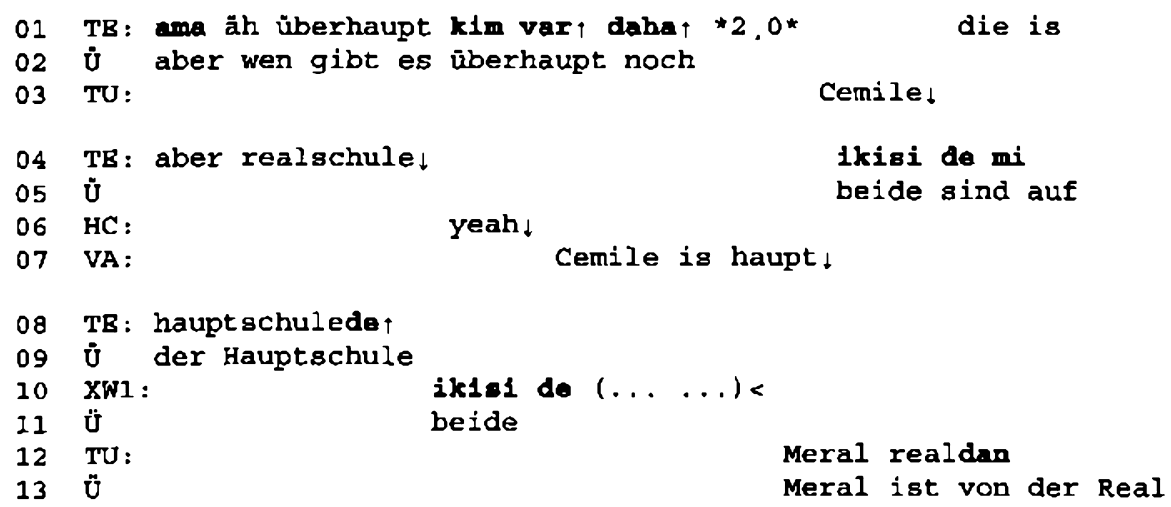


TE :

TU: poye dūp̧tü ya mittlere reifeden $\downarrow$ * bvja

Û doch runter von der mittleren Reife aufs BVJ

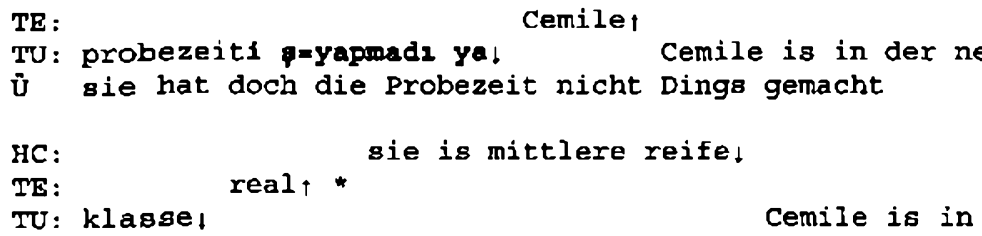

Teslimes Formulierung die is aber realschule (01/04) und Hicrans Formulierung sie is mittlere reife (20) zeigen ghettosprachliche Merkmale. Sie alternieren mit Turas grammatisch korrekten Formulierungen Cemile is in der neuntn klasse (18/22) und Cemile is in der Sickinga hauptschule (22/23). Die Verwendung von Kurzformen für Schulbezeichnungen zusammen mit Lokal- bzw. Richtungsangaben ohne Artikel und Präposition ist typisch für Ghettodeutsch. In der Ingroup-Kommunikation der jüngeren „Powergirls" halten sich solche Spuren des Ghettodeutsch, auch nachdem sie in Schulen außerhalb des Ghettos gewechselt sind. Sie machen die Mädchen für Deutschsprachige auffallend und signalisieren einen „Ghettohintergrund".

Ghettosprachliche Merkmale verschwinden jedoch mit der zunehmenden Intensität deutschsprachiger Kontakte. Bei der älteren Schwester von Teslime, die das Gymnasium abgeschlossen hat, kommen sie nicht mehr vor:

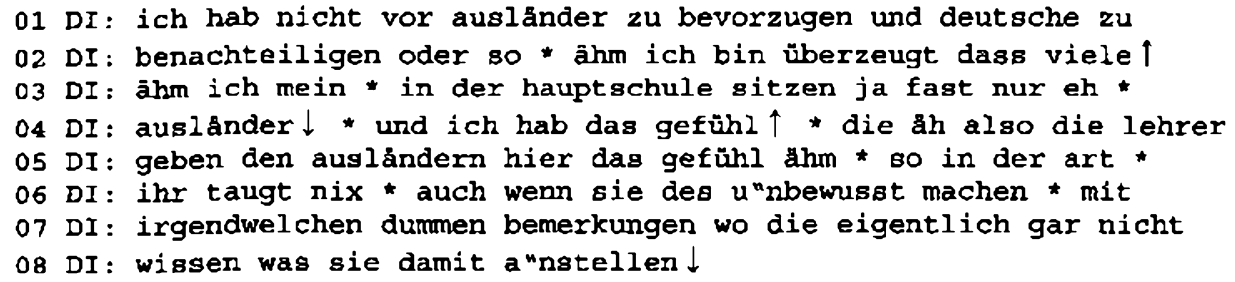

Wie die Beispiele zeigen, verschwinden die grammatischen Merkmale des Ghettodeutsch auf dem Weg aus dem Ghetto und Umgangsdeutsch wird zur Normalität. Doch die Besonderheiten des Ghettodeutsch im prosodisch-phonologischen Bereich bleiben erhalten. Sie kommen auch bei den Sprecherinnen vor, die ein grammatisch einwandfreies Deutsch sprechen. Diese Besonderheiten werden von deutschen Informantinnen als "gestoßenes" Sprechen bezeichnet, ${ }^{42}$ das eine Herkunft aus dem Ghetto signalisiert. Konstitutive Merkmale sind: ${ }^{43}$

42 Ich spielte Ausschnitte aus den Interviews mit den Migrantenjugendlichen Mannheimer Studierenden vor und ließ sie ihren Eindruck beschreiben. Sie bezeichneten die Art des Sprechens als ,aggressiv" und ,gereizt", als ,hervorgestoßen" und als ,sprechen wie aus der Maschinenpistole". Mit dieser Art des Sprechens verbanden sie Sprecherinnen, die ,aus dem Jungbusch" (=das Ghetto) kommen.

43Für Anregungen bei der Identifizierung und Beschreibung dieser Merkmale danke ich Nina Behrend. 
- Tendenz zur Kürzung langer Vokale, wodurch sich auch die Qualität der umgebenden Konsonanten ändert;

- Tilgung des initialen Vokals bei Personalpronomen ich und Fortisierung des [J]-Lauts in Kombinationen wie z.B. $s c h=h a b$ oder $h a h=s c h, m u s s=s c h$;

- Spannung und Fortisierung der stimmlosen Plosive $[\mathrm{p}, \mathrm{t}, \mathrm{k}]$ und Frikative [s, $\left.\mathrm{f}, \int\right]$ vor allem in initialer Position

- deutliche Aspirierung von stimmlosen Plosiven

- deutliche Verstimmhaftung von stimmhaften Plosiven und zusätzliche Verstimmhaftung von Sonoranten

- Fast-Tilgung des /r/ nach kurzen Vokalen;

- hohes Sprechtempo und ein insgesamt gespanntes Sprechen

- Tendenz zu einer „hämmernden" Rhythmisierung durch Gleichbehandlung von betonten und unbetonten Silben, silbenzählender Rhythmus

- die Tendenz zu planer Intonationskontur ohne große Tonhöhenbewegungen.

Die Kookkurrenz dieser Merkmale ist konstitutiv für das Sprechen der Powergirls und für viele Ghettojugendliche. Vor allem die Fortisierung und Längung der Frikative und der hämmernde Rhythmus, machen den scharfen, zischenden Charakter aus. Dadurch unterscheidet sich das Sprechen der Migrantenjugendlichen sehr deutlich von dem „Mannheimerischen", das eine intervallreiche Intonationskontur mit zwei- und dreigipfliger Endkadenz hat und von Mannheimer Sprecherinnen als „breite" und „verwaschene" Sprache bezeichnet wird. ${ }^{44}$ Und dieser typische „Ghetto-Akzent" bleibt erhalten und ist auch in abgeschwächter Form für Mannheimer erkennbar. Entsprechende Erfahrungen schildern die erwachsenen „Powergirls", die nach der Heirat Wohnungen außerhalb des Stadtgebiets suchten. Sie wurden am Telefon, ohne dass sie ihre Namen genannt hatten, nur aufgrund ihrer Sprechweise als „Ausländerinnen" oder „Türkinnen" erkannt und als potentielle Mieterinnen abgewiesen.

\section{Sprach- und Kommunikationspraktiken der schulisch und beruflich Erfolg- reichen: die „Powergirls"}

Die Migrantenjugendlichen, die den Weg aus dem Ghetto schaffen, stellen im untersuchten Stadtgebiet eine kleine Minderheit dar. Es sind vor allem Mädchen, die das Durchhaltvermögen und die enorme Anstrengung aufbringen, um höhere Bildungsabschlüsse zu erreichen. Sie begreifen oft sehr früh, dass der Weg aus der traditionellen türkischen Frauenrolle nur über eine gute Berufsausbildung führt, die sie als Voraussetzung für ein eigenständiges und nicht-familienbestimmtes Leben betrachten.

Beispielhaft für eine solche Entwicklung sind die „Powergirls". ${ }^{45}$ Sie sind zwischen 15 und 22 Jahre alt, Kinder ehemaliger türkischer Gastarbeiter, in dem untersuchten Stadtgebiet aufgewachsen und zur Grundschule gegangen. Einige Mädchen konnten in der 5. Klasse auf weiterfuihrende Schulen außerhalb des Ghettos wechseln, die anderen gingen

44 Vgl. dazu ausführlich Keim 1995, Kap. 3

45 Vgl. dazu ausführlich Keim in Vorb. Teil IL Darin wird die biographische Entwicklung der Powergirls nachgezeichnet und der äußerst schwierige Weg aus dem Ghetto im Detail beschrieben. 
in die Ghettohauptschule und schafften nach dem Abschluss den Übergang auf Fachoberschulen. Die Mädchen schlossen sich als „Powergirls" zusammen, einerseits in Opposition zu dem Leitbild der traditionellen türkischen Frau, die ganz auf die Familie und deren Interessen ausgerichtet ist, und andererseits in Abgrenzung und Opposition zu Deutschen (Mitschülerinnen und Lehrerinnen), durch die sie sich abgelehnt und negativ bewertet fühlen. Die Mädchen leben in der ständigen Spannung zwischen der traditionell geprägten türkischen Migrantengemeinschaft, deren Normen und Werte in starkem Kontrast zu den Anforderungen und Erwartungen stehen, die ihnen in der neuen schulischen Umwelt entgegengebracht werden (zuhause ist Türkei, draußen ist Deutschland). Außerhalb des „Ghettos" erleben sie sehr schmerzlich das negative Fremdbild, das ihnen mit Bezeichnungen wie Scheiß Ausländer oder dreckige Türkin entgegengebracht wird. Nach intensiven Auseinandersetzungen mit solchen Erfahrungen und durch die tatkräftige Unterstützung einer deutsch-türkischen Pädagogin, die für die Mädchen zum Vorbild wurde, haben sie ein neues Selbstverständnis entwickelt: Sie begreifen ihr Leben in Deutschland als Chance für den sozialen Aufstieg und für den Ausbruch aus traditionellen türkischen Leitbildern für Frauen.

\subsection{Sprachrepertoire der „Powergirls"}

Die Mädchen haben im Laufe ihrer schulischen und sozialen Entwicklung ein weites und ausdifferenziertes Sprach- und Kommunikationsrepertoire ausgebildet, das von Umgangsdeutsch, über deutsch-türkische Sprachmischungen bis zu dialektalem Türkisch reicht. Es gibt für alle „Powergirls" Kontexte, in denen entweder nur Deutsch, Türkisch oder Mixing gesprochen wird. Bei Türkisch unterscheiden die Mädchen zwischen dem „Mannheimer Türkisch" mit vielen deutschen Insertionen und Formeln ${ }^{46}$ und dem „Türkisch in der Türkei". Während sie das „Mannheimer Türkisch" gut können, macht ihnen das Türkische in der Türkei Schwierigkeiten: Sie fallen auf, weil sie viele Wörter nicht verstehen und es ihnen schwer fällt, längere Zeit nur Türkisch zu sprechen.

Die Powergirls, die Abitur oder Fachabitur, eine gehobene Berufsausbildung oder ein Studium erreicht haben, beherrschen Standarddeutsch. Für die älteren Mädchen ist es auch in der Ingroup-Kommuniation eine wichtige Kommunikationsform. Ich gebe ein kurzes Beispiel von einer 17jährigen Gymnasiastin, die über ihre Erfahnungen mit deutschen Lehrern spricht:

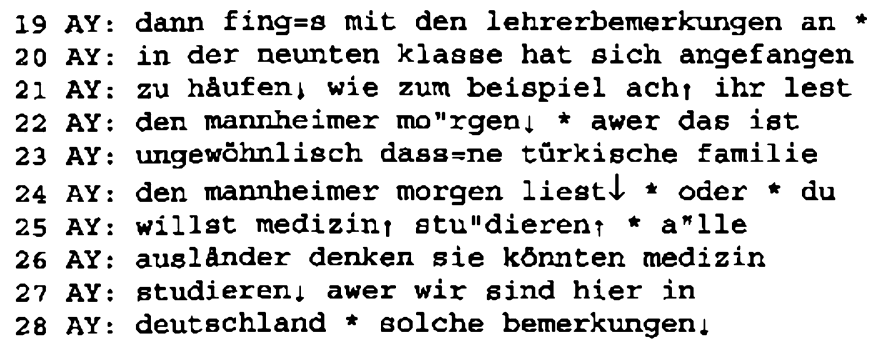


Außerdem haben die Mädchen unter Rückgriff auf Varietäten des Deutschen und des Türkischen spezifische Variationsmuster herausgebildet, die zum symbolischen Verweis auf soziale Kategorien und auf soziale Kontexte dienen. In beiden Bezugsgesellschaften gibt es für die Mädchen zentrale Kategorien, zu denen sie sich in maximalen Kontrast setzen und die sie stark negativ bewerten:

12In der türkischen Migrantengemeinschaft die Kategorie des „Assi"-Türken: Dazu gehören Angehörige der ersten Migrantengeneration, die eng traditionell leben, nur wegen des Geldes in Deutschland sind, Deutsche und Deutsch ablehnen und für ein besseres Leben in der Türkei sparen. Auf Angehörige dieser Kategorie wird durch „Gastarbeiterdeutsch" verwiesen. Das sogenannte „Gastarbeiterdeutsch" ist das ungesteuert erworbene Deutsch der ersten Migrantengeneration, oft pidginisierte und fossilisierte Varietäten des Deutschen. „Gastarbeiterdeutsch" ist nicht die Sprache der Mädchen und mit seiner Verwendung wird immer eine zusätzliche Bedeutungsdimension kontextualisiert. ${ }^{47}$ Außer zur Karikatur des „Assi"-Türken wird es auch eingesetzt, wenn die Mädchen sich in Gegenwart von Deutschen von ihren Eltern und deren schlechten Deutschkenntnissen distanzieren. Dabei werden die Eltern für einen kurzen Moment in die Nähe der Kategorie des „Assi"-Türken gebracht, einer Kategorie, von der sie die Eltern in anderen Kontexten explizit ausnehmen.

13In der Mehrheitsgesellschaft die Kategorie „des dummen Deutschen": Angehörige dieser Kategorie halten sich für „besser" als die Mädchen, sind ihnen gegenüber feindselig, werten sie ab, sind engstirnig, fantasielos und selbstzufrieden. Zum symbolischen Verweis auf Angehörige dieser Kategorie verwenden die Mädchen breites „Mannheimerisch", wie es in ihrem lokalen Umfeld von deutschen Einheimischen gesprochen wird. Das Deutsch der "Powergirls" ist zwar leicht regional gefärbt ${ }^{48}$ ist jedoch insgesamt standardnah geprägt. Formen des Mannheimerischen gehören nicht zur unmarkierten Ingroup-Kommunikation und kontextualisieren ebenfalls eine zusätzliche Bedeutung:49 „Mannheimerisch" wird zur Karikatur des "dummen" und ausländerfeindlichen Deutschen verwendet und hat für die Mädchen darüber hinaus auch die Bedeutung eines „fun-codes"; d.h. es dient in der Kommunikation untereinander zur Signalisierung von Ironie und Spiel.

\subsection{Deutsch-türkisches Mixing}

Zentrales Kommunikationsmittel in der Ingroup-Kommunikation der „Powergirls" ist deutsch-türkisches Mixing, das die Mädchen in elaborierter Form ausgebildet haben. ${ }^{50}$ Für diese Kommunikationspraxis haben sie eine eigene Bezeichnung; sie nennen sie "Mischmasch" oder „Mixmax". Mixing wird mit hoher Fertigkeit und Sprechgeschwindigkeit produziert und die Wechsel finden nach durchschnittlich jedem 5.- 6. Wort statt. Es gibt mehr inter-clause als intra-clause Wechsel und die deutschen und türkischen Segmente im Mixing sind nach den Regeln der jeweiligen Sprache konstruiert.

47 Beispiele und Analyse der Funktionen von Gastarbeiterdeutsch in Keim 2002a

48Z.B. durch tags wie gell und weeschd (weißt du) oder durch Verschleifungen wie ham =ma (haben wir), $s i=m a$ (sind wir) u.ä.

49 Vgl. dazu Keim 2002b und Kallmeyer/ Keim 2003b.

50 Einen Beitrag zur Beschreibung des Mixing liefert Y. Balci in diesem Heft, die die deutschen Insertio nen semantisch analysiert. 
In der Ingroup-Kommunikation gibt es kommunikative Aktivitäten oder Genres, die ausschließlich in Mixing produziert werden; d.h. für diese Genres ist Mixing konstitutiv. Das sind vor allem Erzählungen und Auseinandersetzungen. Dabei dienen Mixingmuster zur Strukturierung von Informationen und zur Konturierung und Fokussienung von diskursiven Einheiten, zur Unterscheidung und Kontrastierung von Perspektiven. Das werde ich im Folgenden ausführlich darstellen.

\subsubsection{Mixingmuster in Erzählungen. ${ }^{51}$}

Für Erzählungen gibt es stabile Mixingmuster: Das Erzählgerüst ist in Türkisch, Reaktionen darauf und Kommentare dazu sind in Deutsch; szenische Darstellungen werden durch Sprachwechsel gerahmt. An einem kleinen Beispiel möchte ich diese Muster verdeutlichen. Eines der Mädchen erzählt über ein Schulprojekt, bei dem die Mädchen Erfahrungen eines Behinderten machen sollten. Dazu setzte sich eines der Mädchen in einen Rollstuhl und wurde von einem anderen durch die Stadt geschoben. Die Erzählung besteht aus einer Aneinanderreihung von Episoden, in denen die Erzählerin einzelne Ereignisse darstellt, die sie auf ihrem Rundweg im Rollstuhl erlebte.

a) Türkische Elemente (Konnektoren, Partikel) werden verwendet, um den Aktivitätstyp „Erzählen" ebenso wie einzelne Erzählepisoden zu rahmen und vom vorangehenden bzw. nachfolgenden deutschen Kontext (Kommentare, Diskussionen, u.ä.) abzugrenzen. In unserem Beispiel sind die Ankündigung der Erzählung, ihre Ratifizierung und die Orientierungssequenz in Deutsch formuliert; der Beginn der ersten Episode ist durch ein türkisches Element markiert:

[Die Interaktion vor dem Transkriptausschnitt ist in Deutsch]

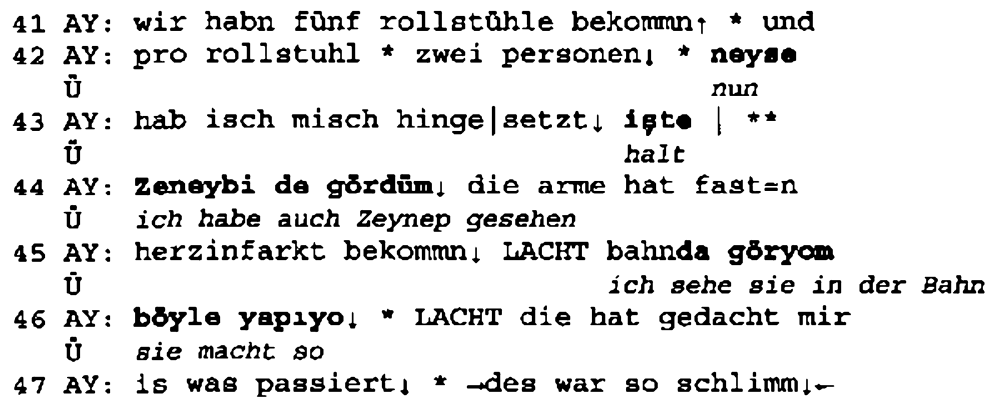

Die Erzählung und die erste Episode beginnt mit dem narrativen türkischen Konnektor neyse (nun) und das erste Erzählsegment endet mit der türkischen Partike iște (halt).

D.h. das erste Erzählsegment ist durch türkische Elemente gerahmt und führt Mixing als Mittel der Darstellung ein. 
b) Handlungen und Perspektiven verschiedener Akteure werden durch Sprachwechsel kontrastiert, im Beispiel die Perspektive der Erzählerin und die ihrer Freundin Zeynep:

- Die Darstellung der Perspektive der Erzählerin erfolgt in Türkisch:

44 AY: Zeneybi de gōrdûm $\downarrow$

Uี ich habe Zeynep gesehen

- Die Darstellung der Reaktion der Freundin, die über die Erzählerin im Rollstuhl er schrickt, erfolgt in Deutsch:

$45 \mathrm{AY}$ : die arme hat fast=n herzinfarkt bekomm

Dasselbe Sprachwechselmuster wird direkt im Anschluss verwendet, bei der Reformulierung der kurzen Szene:

- Die Darstellung der Perspektive der Erzählerin erfolgt wieder in Türkisch:

45 AY: LACHT bahnda göryom bbyle yapıyol *

Ü Ich sehe sie in der Bahn sie macht 80

- Die Darstellung der Perspektive der Freundin ist in Deutsch:

46 AY: LACHT die hat gedacht mir is was passiert $\downarrow$ *

So wie in dieser ersten Erzählepisode werden auch in den folgenden Episoden die Handlungen und Erfahrungen der Erzählerin in Türkisch dargestellt; die Reaktionen der anderen, im Beispiel die der Freundin, in weiteren Episoden die Reaktionen deutscher Passanten auf der Straße, sind in Deutsch formuliert.

c) Zitate sind in der Sprache, in der die zitierten Personen in der Referenzsituation gesprochen haben. Die Zitate sind gerahmt durch sprachlich kontrastierende Zitatein leitungen oder Schlussformeln. Dieses Muster kann in einer weiteren Episode de monstriert werden:

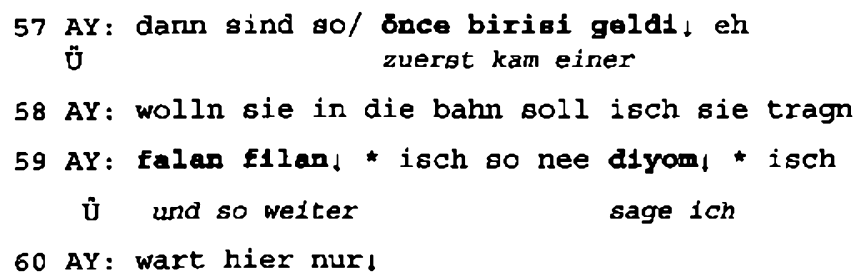

Die Leute, die die Erzählerin auf ihrer Tour durch die Stadt traf, waren Deutsche, d.h. die Gespräche fanden in Deutsch statt. So werden sie auch in der Erzählung präsentiert: Die Frage des Mannes, den die Erzählerin traf, ist in Deutsch: wolln sie in die Bahn soll isch sie tragn (58), ebenso wie ihre Antwort auf die Frage: nee (59). Das Zitat des Mannes wird mit einer türkischen Formel beendet falan filan und das Eigenzitat mit einer deutschen Formel eingeleitet isch so und einem türkischen Element beendet diyom; d.h. der Dialog mit dem Mann auf der Straße ist durch türkische Elemente gerahmt, die die Zitate einleiten oder ausleiten. Die Szene hat folgende Struktur: 
Zitat des Mannes: wolln sie in die bahn soll isch sie tragen falan filan

\section{Zitat}

$\begin{array}{lllll}\text { Eigenzitat: } & \text { isch so } & \text { nee } & \text { diyom } & \text { isch wart hier nur } \\ & \text { Einleitung } & \text { Zitat } & \text { Aus-/Einleitung } & \text { Zitat }\end{array}$

In diesem Beispiel kann auch ein weiteres Muster gezeigt werden: Beginn einer Episode ist in Türkisch. Die Stabilität dieses Muster wird besonders durch die Selbstkorrektur der Erzählerin deutlich, mit der sie das Muster etabliert: Sie beginnt die Episode in Deutsch, bricht ab und reformuliert den Episodenbeginn in Türkisch:

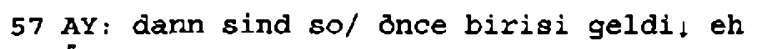

Mit der Selbstkorrektur direkt nach dem Episodenstart und dem Sprachwechsel ins Türkische hebt die Erzählerin das zugrunde liegende Muster hervor und zeigt seine Stabilität.

d) Es gibt zweiteilige Strukturen der Informationsverarbeitung mit einem festem Variationsmuster: der erste Teil (z.B. Handlungsdarstellung, Hintergrund, Voraussetzung u.ä.) erscheint in Türkisch, der zweite Teil (Detaillierung/ Präzisierung, Vordergnund, Folge u.ä.) erscheint in Deutsch. Im folgenden Beispiel folgt auf die Handlungsdarstellung in Türkisch: adam kapıyı açıp tutyo (der Mann hält die Tür auf) die Detaillierung in Deutsch: der steht so an der wand. In einem anderen Beispiel erscheint die Voraussetzung in Türkisch: sonra yoruldum (dann bin ich müde geworden), die Folge in Deutsch: isch bin grad so stehen geblieben. Solche zweiteiligen Strukturmuster mit fester Zuordnung zwischen Sprachen und Funktionen sind charakteristisch für das Mixing der „Powergirls". Sie sind darüber hinaus auch bei Angehörigen der zweiten und dritten Migrantengeneration im Ghetto weit verbreitet, wie die oben (S. 207) dargestellte Mixingpraxis des 6jährigen Jungen zeigt.

\subsubsection{Mixingmuster in Auseinandersetzungen}

In Auseinandersetzungen folgen die „Powergirls" ganz allgemein den in Mehrsprachigkeitssituationen beobachteten und beschriebenen Prinzipien von Konvergenz und Divergenz. Sprachliche Konvergenz bedeutet: Wenn der zweite Sprecher mit dem ersten Sprecher übereinstimmt, übernimmt er seine Sprache. Die Sprachübernahme verstärkt die inhaltliche Übereinstimmung. Die Sprachwechselmuster sehen folgendermaßen aus:

a) AA: Deutsch

BB: Deutsch

b) AA: Türkisch

$\mathrm{BB}$ :

Türkisch

Sprachliche Divergenz liegt vor, wenn der zweite Sprecher nicht mit dem ersten übereinstimmt oder ihm widerspricht und dabei in die andere Sprache wechselt. Durch den Sprachwechsel hebt er die inhaltliche Opposition hervor. Die Sprachwechselmuster sehen in diesem Fall folgendermaßen aus: 
c) AA: Deutsch

$\mathrm{BB}$

Türkisch

d) AA: Türkisch

$\mathrm{BB}$ :

Deutsch

Diese Sprachwechselmuster können im folgenden Gesprächsbeispiel gezeigt werden. Zwei Mädchen, Gülsen (GL) and Selma (SE), streiten darüber, wer die kleine Schwester zuhause abholen und mit ihr zum Arzt gehen soll. Vor dem Transkriptausschnitt fordert Gülsen Selma auf, die kleine Schwester zu holen; das formuliert sie in Türkisch. Selma weigert sich; dabei spricht sie Deutsch. Der folgende Ausschnitt beginnt mit Gülsens Aufforderung, dass Selma zuhause anrufen soll, um zu sagen, dass sie nicht kommen kann:

[Deutscher Beitrag von SE]

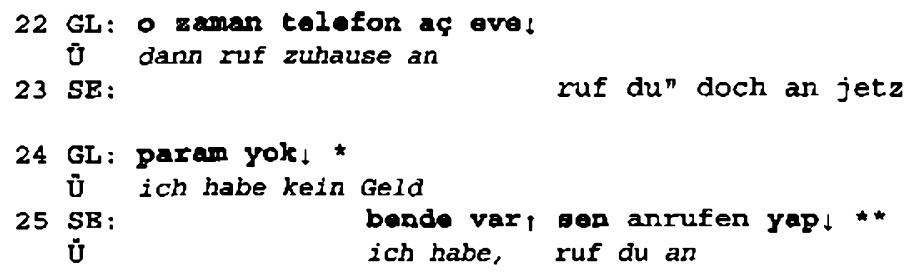

Gülsens Aufforderung ist in Türkisch o zaman telefon aç eve $\downarrow$ (dann ruf zuhause an, 22) und kontrastiert zu dem vorangehenden deutschen Redebeitrag Seimas. Selma weist die Aufforderung durch eine Retourkutsche zurück und wechselt dabei ins Deutsche: $r u f d u$ " doch an jetz (23). Auf Seimas Widerspruch reagiert Gülsen ebenfalls mit einer Verweigerung und behauptet, dass ihr eine wichtige Voraussetzung fehlt, um der Forderung nachzukommen: param yok (ich hab kein Geld, 24). Die Zurückweisung ist wieder mit Sprachwechsel verbunden. Im letzten Beitrag ändert sich das Sprachwechselmuster: Selma bietet eine Problemlösung an und schlägt vor, Gülsen Geld zu leihen, damit sie anrufen kann. Selma kooperiert mit Gülsen und hebt die Zusammenarbeit durch sprachliche Konvergenz hervor: Sie spricht Türkisch und übernimmt die Sprache, die Gülsen in dem vorherigen Beitrag verwendet hat. Die gesamte Interaktion hat folgendes Muster:

$\begin{array}{ll}\text { GL: erste Forderung (die Schwester abholen) } & \text { Türkisch } \\ \text { SE: Zurückweisung } & \text { Deutsch } \\ \text { GL: zweite Forderung (zuhause anrufen) } & \text { Türkisch } \\ \text { SE: Retourkutsche } & \text { Deutsch } \\ \text { GL: Zurückweisung (kein Geld) } & \text { Türkisch } \\ \text { SE: Lösungsangebot (Geld leihen) } & \text { Türkisch }\end{array}$

In dieser Interaktionssequenz werden beide Sprachwechselmuster verwendet; in den ersten Beiträgen das Muster zur Hervorhebung von Widerspruch mit Sprachwechsel und in dem letzten Beitragspaar das Muster für Kooperation mit der Übernahme der Sprache der Vorrednerin. 


\subsection{Kommunikationspraxis und Selbstdefinition}

Die elaborierten Wechselmuster, die die „Powergirls" entwickelt haben, weisen darauf hin, dass, obwohl die Mädchen eine hohe Kompetenz in Deutsch haben, Mixing eine hoch bewertete Kommunikationsform ist, und die Mädchen es als eigene Ausdrucksform verstehen und kultivieren. Keine der anderen Migrantengruppen, die wir im Rahmen unseres Projektes untersuchten, hat solch elaborierte Mixingmuster entwickelt. ${ }^{52}$ Die Bevorzugung von Mixing und die Arbeit daran, stehen in engem Zusammenhang mit der Selbstpositionierung der „Powergirls" in Relation zu den beiden Bezugsgesellschaften: einerseits zur tendenziell monolingualen türkischen Migrantengemeinschaft, die von den Mädchen Loyalität zu ihren Traditionen, Normen und Werten fordert; und andererseits zur monolingualen Mehrheitsgesellschaft, von der sie sich abgelehnt und abgewertet fühlen. Die „Powergirls" wollen zu keiner der beiden Bezugsgesellschaften gehören, sie distanzieren sich von den Anfordenungen beider und entwickeln ein neues, hybrides Selbstverständnis: Sie verstehen sich als weder deutsch noch türkisch, sondem als etwas „Neues", als junge Deutsch-Türkinnen. Mixing ist das Symbol für dieses neue Selbstverständnis, wie eine der jungen Frauen das ausdrückt:

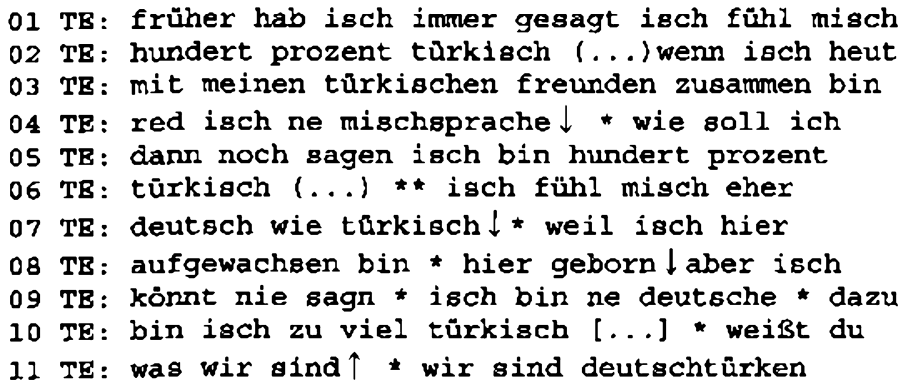

Für die Sprecherin stehen Sprache und ethnische Zugehörigkeit in einem sehr engen Zusammenhang, und die Bevorzugung einer bestimmten Kommunikationspraxis mit Freunden und Vertrauten ist für sie Ausdruck ihrer kulturellen Zugehörigkeit. Für sie indiziert die Bevorzugung von Mixing in engen freundschaftlichen Beziehungen die Nichtzugehörigkeit zur deutschen oder zur türkischen Gruppe und die Zugehörigkeit zu einer Kategorie jenseits von Deutsch- oder Türkischsein: zu der neuen hybriden Kategorie der „Deutschtürkin". Diese Kategorie ist - im Gegensatz zu den vorgegebenen ethnischen Kategorien "deutsch" und "türkisch" - positiv bewertet, und mit ihr verbinden die „Powergirls" ein besonderes, kreatives Potential:

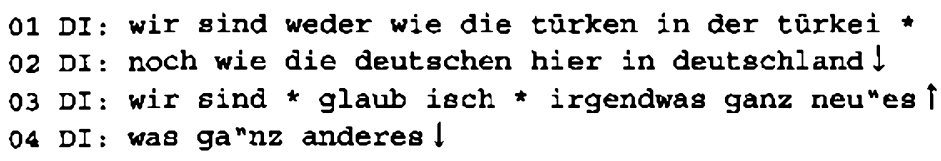

Die Verwendung von Mixing als Ingroup-Code und als Code, mit dem enge Beziehungen und Intimität ausgedrückt werden, schließt monolinguale Deutsche und Türken von

Vgl. die Untersuchungen zur Gruppe der „Unmündigen" von I. Cindark und zu den Europatürken von S.

Aslan, in diesem Band. 
engen Freundschaften mit den „Powergirls" aus. Mixing hat für die Mädchen eine derart große Bedeutung, dass es sogar als zentrales Element für die Definition eines zukünftigen Lebenspartners dient. Das beschreibt eines der Mädchen folgendermaßen:

01 FU: isch könnte nie einen mann lieben * der meine

02 FU: sprache nischt spricht $\downarrow$ die minschsprache $\downarrow$ *

03 FU: einen tūrken nischt und auch keinen deutschen

\section{Ausblick}

Die Entwicklungskarrieren der „Powergirls" zeigen, dass auch aus dem „Ghetto" heraus die Ausbildung eines eigenständigen Selbstbildes und der Erwerb sozialer und kommunikativer Kompetenzen gelingen können, die die gleichberechtigte Teilhabe an zentralen Bereichen der Mehrheitsgesellschaft ermöglichen. Doch um das zu erreichen, brauchen die Kinder Unterstützung und vor allem positive Leitbilder, die ihnen Identifikationsangebote machen. ${ }^{53} \mathrm{Um}$ die Lebenschancen der Ghettokinder zu verbessem, müssen ihre Fähigkeiten besser erkannt und ihr schulischer Erfolg muss drastisch erhöht werden. Die Bilingualität der Kinder darf von Schule und Öffentlichkeit nicht länger als Problem gesehen, sondem muss als gesellschaftliche Ressource aufgewertet und ausgebaut werden. Den Kindern müssen Gefühle der Unterlegenheit und Erfahrungen des schulischen Scheiterns erspart werden. Sie müssen die Chance haben in der Mehrheitsgesellschaft sozialen und beruflichen Erfolg zu erreichen und gleichzeitig - jenseits vorgegebener ethnischer Kategorien - eigenständige sozial-kulturelle Identitäten und neue Kommunikationsformen auszubilden, wie das die „Powergirls" geleistet haben. Dazu sind gezielte Fördermaßnahmen auf allen Bildungsstufen, eine Verbesserung des Angebots in den Bildungsinstitutionen ebenso wie eine breite Aufklärung der deutschen Offentlichkeit und der Migrantengruppen notwendig. Um der weiteren Desintegration und Abschottung von Ghettojugendlichen entgegenzuwirken, müssen anti-schulische und anti-deutsche Haltungen aufgebrochen und verändert werden. Vor allem aber muss in der Öffentlichkeit ein Bewusstsein dafür geschaffen werden, dass Migrantenkinder und ihre Familien deutliche Signale von Seiten der Deutschen brauchen, dass sie angenommen und respektiert werden, damit sie auch ihrerseits die Anstrengungen aufbringen können, die eine erfolgreiche berufliche und soziale Entwicklung erforderlich macht.

53 Besonders wirksam sind positive Leitbilder aus der eigenen Population: Im Fall der „Powergirls" ist das eine sehr selbständige, selbstbewusste und kompetente bilinguale deutsch-türkische Pädagogin, die großen Einfluss auf die positive Entwicklung der Mädchen hatte; im Falle der männlichen türkischen-stämmigen Jugendlichen, die Zifonun/Cindark in diesem Band beschreiben, ist es ein türkischer Fuss-ballverein, der ihnen positive Identifikationsangebote macht und ihre soziale Integration beeinflusst und voranbringt. 


\section{Transkriptionskonventionen}

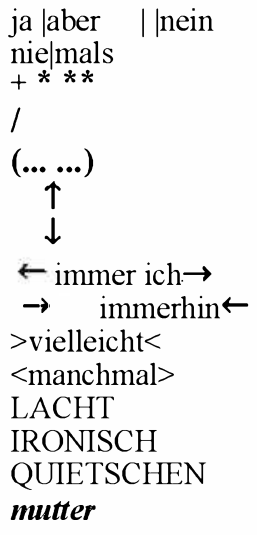

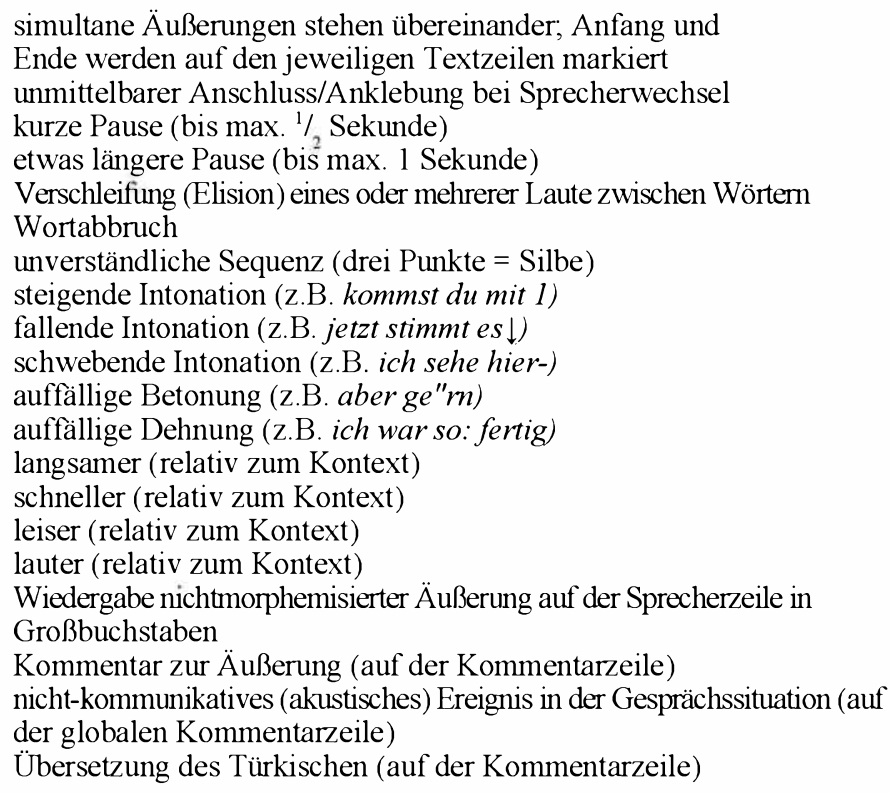

\section{Literatur}

Androutsopoulos, Jannis (2001): Ultra korregd Alder! Zur medialen Stilisienung und Aneignung von Türkendeutsch'. Deutsche Sprache 29, Heft,4, S. 321-339.

Aslan, Sema (i.Vor.): Sprach- und Kommunikationsverhalten türkischer Kinder im Kindergarten und in der Grundschule. Dissertationsarbeit. Mannheim

Auer, Peter (ed.) (1998): Code-switching in conversation. London: Routhledge.

Auer, Peter (2003): Türkenslang' - ein jugendsprachlicher Ethnolect des Deutschen und seine Transformationen. In: Buhofer-Häcki, Annelies (ed.): Spracherwerb und Lebensalter. Tübingen/ Basel: Francke, S. 255-264.

Backus, Ad (1996): Two in one: Bilingual Speech of Turkish migrants in the Netherlands. Tilburg: University Press.

Bierbach, Christine \& Gabriele Birken-Silverman (2002): Kommunikationsstil und sprachliche Symbolisierung in einer Gruppe italienischer Migrantenjugendlicher aus der HipHop-Szene in Mannheim. In: Inken Keim/ Wilfried Schütte (Hgg.): Soziale Welten und kommunikative Stile. Tübingen: Narr, S. 187-215.

Cindark, Ibrahim \& Aslan, Sema (2004): Deutschlandtürkisch? Online. http.V/www.prag. soziostilistik/Deutschlandtuerkisch.pdf. 08.07.2004 MS

Cindark, Ibrahim (i.Vorb.): Die Unmündigen - der kommunikative soziale Stil der emanzipierten Migranten. Dissertationsarbeit.

Dirim, Inci (1998): Var mi lan Marmelade? - Türkisch-deutscher Sprachkontakt in einer Grundschulklasse. Münster: Waxmann. 
Dirim, Inci/Auer, Peter (2004): Türkisch sprechen nicht nur die Türken. Berlin/ New York: De Gruyter.

Füglein, Rosemarie (2000). Kanak Sprak. Eine ethnolinguistische Untersuchung eines Sprachphänomens in Deutschland. Diplomarbeit, Universität Bamberg.

Heitmeyer, Wilhelm/Müller, Joachim/Schröder, Helmut (1997): Verlockender Fundamentalismus. Frankfurt: Suhrkamp.

Hinnenkamp, Volker (2005): Zwei zu bir miydi?' - Mischsprachliche Varietäten von Migrantenjugendlichen im Hybriditätsdiskurs. In: Hinnenkamp, Volker/Meng, Katharina (Hg.): Sprachgrenzen überspringen. Sprachliche Hybridität und polykulturelles Selbstverständnis. Tübingen: Narr, S. 51-104.

Jorgensen, J. Norman (1998): Children's acquisition of code-switching for power wielding. In: Auer (ed.), S. 237-261.

Kalimeyer, Werner/Keim, Inken (2003a): Linguistic Variation and the construction of social identity in a German-Turkish setting. A case study of an immigrant youth-group in Mannheim/Germany. In: Androutsopoulos, Jannis \& Alexandra Georgakopoulou, (eds.): Discourse constructions of youth identities. Amsterdam/Philadelphia: Benjamins, S. 29-46.

Kalimeyer, Wemer/Keim Inken (2003b): Eigenschaften von sozialen Stilen der Kommunikation: Am Beispiel einer türkischen Migrantinnengruppe. In: Erfurt, Jürgen (ed.): „Multisprech": Hybridität, Variation, Identität. OBST, 65, S. 35-56.

Werner Kallmeyer/Keim, Inken/Aslan, Sema/Cindark, Ibrahim (2002): Variationsprofile. Zur Analyse der Variationspraxis bei den Powergirls. [Arbeitspapier PDF, $195 \mathrm{k}$ ]

Keim, Inken (1995): Kommunikative Stilistik einer sozialen Welt „Kleiner Leute" in der Mannheimer Innenstadt. Kommunikation in der Stadt. Teil 3. Berlin/New York: De Gruyter.

Keim, Inken (2002a): Sprachvariation und Bedeutungskonstitution. Die Verwendung von Gastarbeiterdeutsch in Gesprächen junger Türkinnen. In: Deppermann, Amulf/Spranz-Fogasy, Thomas (eds.): bedeuten. Wie Bedeutung im Gespräch entsteht. Tübingen: Stauffenberg, S. 134-157.

Keim, Inken (2002b): Die Verwendung von Formen der Mannheimer Stadtsprache in einer jugendlichen Migrantinnengruppe. In: Bateman, John/Wildgen, Wolfgang (eds.): Sprachbewusstheit im schulischen und sozialen Kontext. Frankfurt/Berlin/ New York: Peter Lang, S. 117-137.

Keim, Inken (2003): Social style of communication and bilingual speech practices: Case study of three migrant youth groups of Turkish origin in Mannheim/ Germany. In: Turkic Languages 2003, Heft 6/2, S. 284-298.

Keim, Inken (2004): Linguistic Variation and communicative practices in migrant children and youth groups. In: Jorgensen, Normann (ed.): Bilingual Papers. Kopenhagen, S. 78-94.

Keim, Inken (i.Vorb.): Die „Powergirls" - eine Gruppe türkischstämmiger Migrantinnen in Mannheim. MS Mannheim.

Kelek, Necla (2005). Die fremde Braut. Ein Bericht aus dem Inneren des türkischen Lebens in Deutschland. Köln: Kiepenheuer/Witsch.

Kotsinas, Ulla-Britt (1998): Language contact in Rinkeby, an immigrant suburb. In: Androutsopoulos, Jannis/Scholz, Arno (eds.): Jugendsprache. Frankfurt: Peter Lang, S. 125-148.

Pfaff, Carol (1994): Early bilingual development of Turkish children in Berlin. In: Extra, Guus/Verhoeven, L. (eds.): The cross-linguistic study of bilingual development. Amsterdam: Royal Netherlands Academy of Arts and Sciences,S. 75-97.

Pfaff, Carol (1999): Changing patterns of language mixing in a bilingual child. In: Extra, Guus/Verhoeven, L. (eds.): Bilingualism and Migration. Berlin: Mouton, S. 97-121. 
Poplack, Shana (1980): Sometimes I'll start a sentence in English y termino en espanol. In: Linguistics 18, S. 581-616.

Sirim, Emran (2004): Bilinguales Sprachverhalten bei jungen Deutschtürken. Magisterarbeit. Universität Mannheim.

Tertilt, Hermann (1996): Turkish Power Boys. Ethnographie einer Jugendbande. Frankfurt: Rowohlt. Zaimoğlu Feridun (1995): Kanaksprak. Hamburg: Rotbuch. 